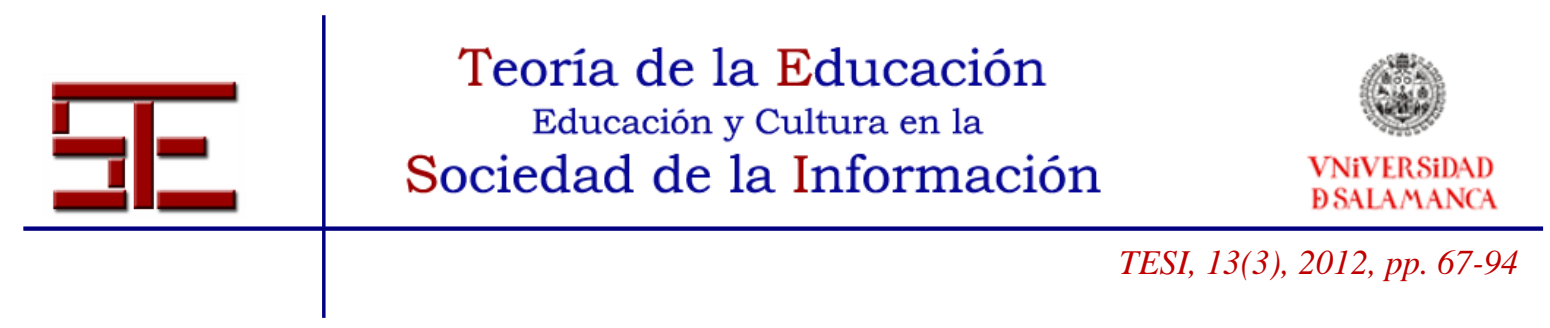

\title{
DETERMINANTES SOBRE LA PERCEPCIÓN CIUDADANA DE LA EDUCACIÓN EN COLOMBIA
}

Resumen: El presente artículo expone los resultados del proyecto "Análisis de los determinantes de la percepción ciudadana sobre la Educación en Colombia“, realizado desde la Universidad del Norte, a partir de la Encuesta Nacional de Percepción Ciudadana realizada por Educación Compromiso de Todos en 2010. El objetivo de este estudio es analizar los principales determinantes que inciden en la percepción ciudadana en materia educativa en Colombia. El trabajo parte de un análisis inferencial, en el que se apreció una mejor valoración de la educación en Colombia cuando existe satisfacción en: la administración de los colegios; la actualización de los profesores; la dotación escolar; el servicio de alimentación escolar; el derecho a la educación; la capacidad de inversión y de gestión local en materia educativa; la utilidad de las pruebas académicas; y cuando se tiene una posición favorable sobre la situación socio-económica del país.

Palabras clave: percepción; educación; Colombia; ciudadanos; determinantes.

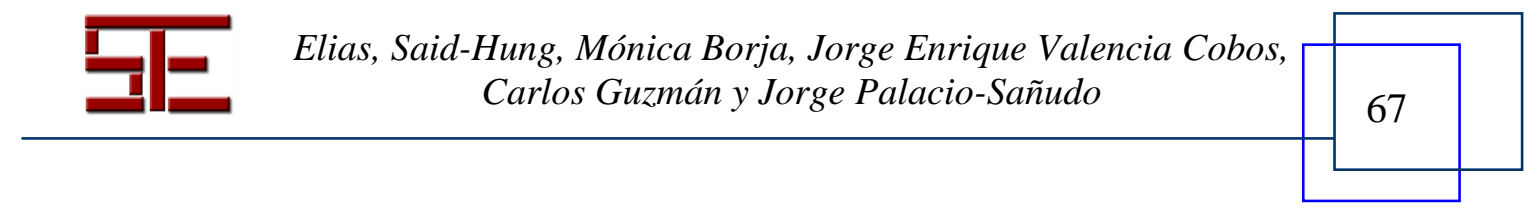




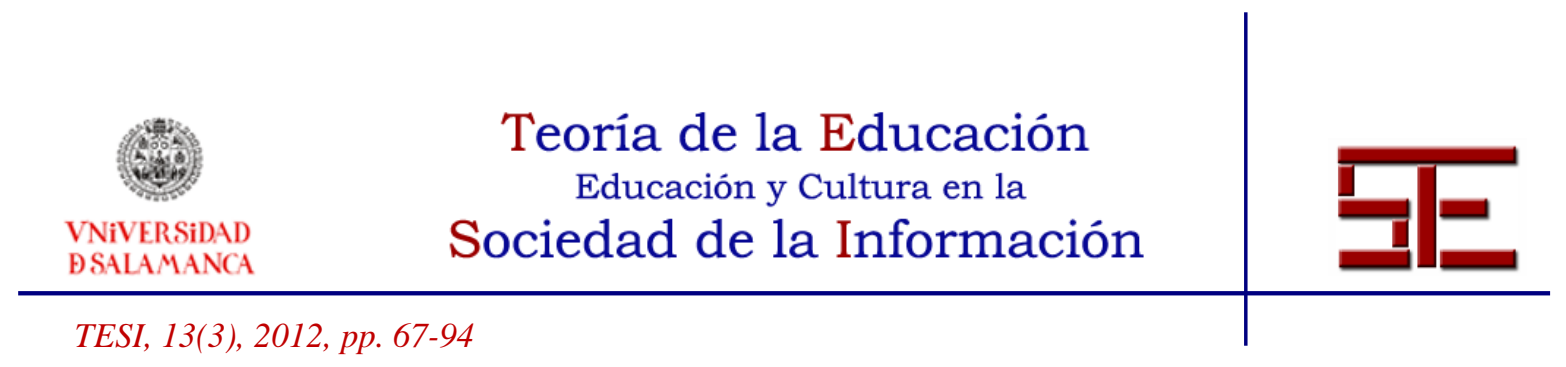

\section{CITIZEN PERCEPTION ABOUT THE EDUCATION IN COLOMBIA}

Abstract: This article presents the results of the Project "Analysis of the determinants of the citizens' perception about education in Colombia" developed by Universidad del Norte from the National Survey of Citizens' Perception developed by Educación Compromiso de Todos in 2010. The objective of this study is to analyze the main determinants influencing the citizens' perception concerning education in Colombia. This work starts with an inferential analysis where a better valuation of education in Colombia was evidenced when there is satisfaction with the administration of schools, teachers' updating, school infrastructure, school food service, the right to education, investment capacity and local management in education, the usefulness of academic tests and when there is a good perception of de socio-economical situation of the country.

Keywords: perception; Colombia; education; citizens; determinants.

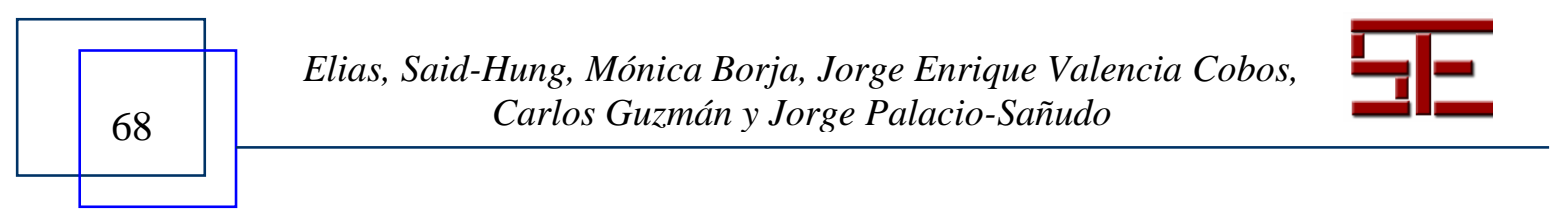




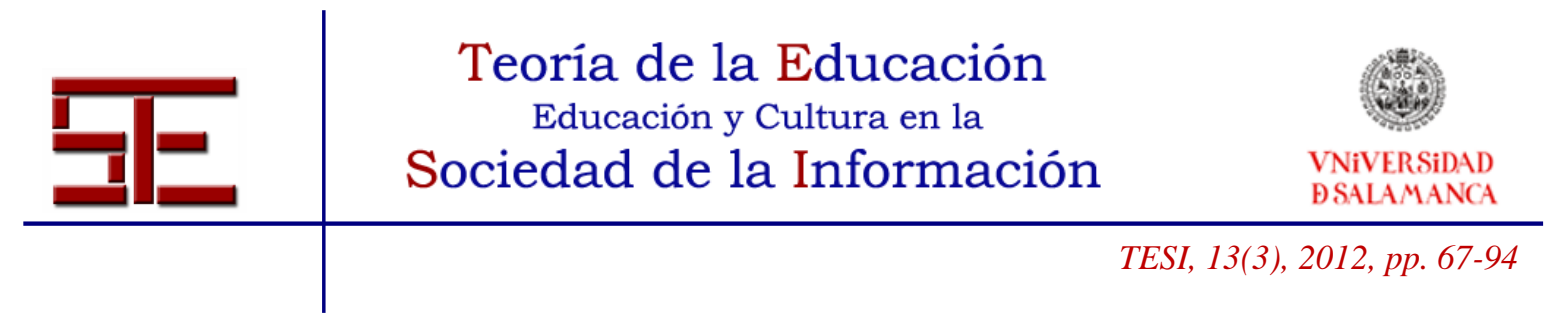

\section{DETERMINANTES SOBRE LA PERCEPCIÓN CIUDADANA DE LA EDUCACIÓN EN COLOMBIA}

Fecha de recepción: 12/12/2011; fecha de aceptación: 18/09/2012; fecha de publicación: 30/11/2012

Elias Said-Hung

saide@uninorte.edu.co

Fundación Universidad del Norte. Colombia

Mónica Borjas

mborjas@uninorte.edu.co

Fundación Universidad del Norte. Colombia

Jorge Valencia Cobos

jorva12911@yahoo.es

Fundación Universidad del Norte. Colombia

Jorge Enrique Palacio-Sañudo

jpalacio@uninorte.edu.co

Fundación Universidad del Norte. Colombia

Carlos Guzmán

cguzmane@uninorte.edu.co

Fundación Universidad del Norte. Colombia

\section{1.- INTRODUCCIÓN}

Hablar de educación es introducirnos en un ámbito complejo, que atraviesa por múltiples elementos: jurídicos, políticos, económicos, sociales, éticos, etcétera y cada uno de ellos participa en lo que se hace, cómo se hace y para qué se hace.

El conocimiento que los ciudadanos tienen en materia educativa depende, fundamentalmente, de sus niveles de instrucción, de sus experiencias en este ámbito, de las relaciones que crean con su entorno social, de las interacciones que establecen con los que actualmente estudian o con los profesionales con quien tienen contacto. Cada uno de estos aspectos les ofrece a los ciudadanos la construcción de un referente de estándares sobre lo que cada uno puede esperar de la educación; así como también, la

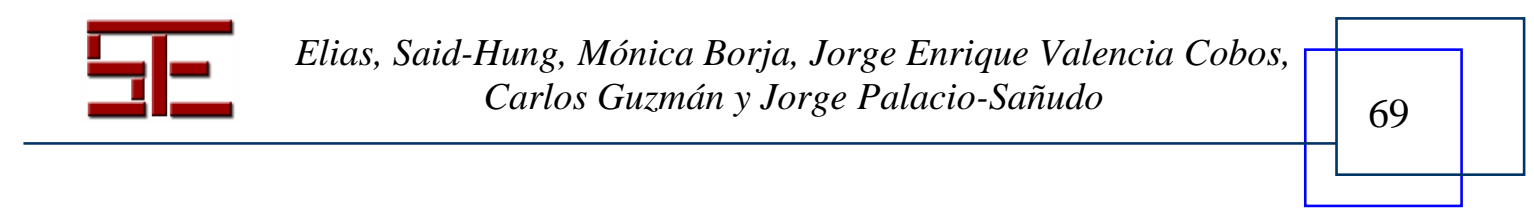




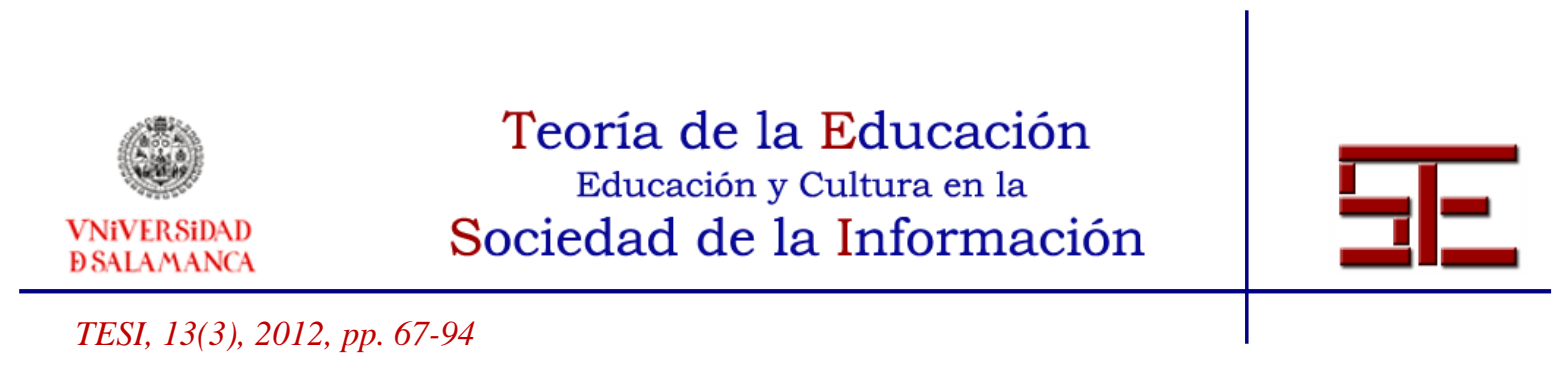

disposición de mecanismos que les ayuden a juzgar los resultados de ésta. Se puede afirmar entonces que la percepción ciudadana sobre la calidad de la educación se deriva de factores sociales e históricos que llevan a consolidar una valoración sobre ella. Frente a esta variabilidad de opiniones se pretende, en este artículo, conocer el nivel de satisfacción de los ciudadanos sobre la educación; de acuerdo con variables sociodemográficas, variables asociadas a la percepción y conocimiento del derecho a la educación, y de variables asociadas a la percepción de los procesos de calidad educativa.

Para los fines descritos en el párrafo anterior, este artículo se centrará en el análisis de los resultados generados en el proyecto "Análisis de los determinantes de la percepción ciudadana sobre la Educación en Colombia“, realizado desde la Universidad del Norte, a partir de la Encuesta Nacional de Percepción Ciudadana realizada en 2010, por Educación Compromiso de $\operatorname{Todos}^{1}$ en Colombia. Ello, con el objetivo de analizar los principales determinantes que inciden en la percepción ciudadana en materia educativa en Colombia, desde el abordaje de cinco dimensiones: la formación docente, la capacidad de gestión al interior de las escuelas, la infraestructura dispuesta para la enseñanza básica y media, el acceso a programas sociales de atención a población en edad de escolarización en Colombia, y la flexibilidad de los programas curriculares dispuestos desde las escuelas; todas estas serán analizadas desde la opinión pública dispuesta en los ciudadanos residentes en Colombia. El objetivo antes expuesto será concretado a partir de la búsqueda de la resolución a las siguientes interrogantes: ¿Cuáles son los factores que inciden en la percepción y en el nivel de satisfacción que tienen los ciudadanos del sistema educativo y de la educación, en general, en Colombia? ¿Cuáles son los aspectos que condicionan la percepción y conocimiento que tienen los ciudadanos en torno al derecho a la educación en el país, así como a la calidad educativa existente en Colombia?

\footnotetext{
${ }^{1}$ http://www.educacioncompromisodetodos.org/ect/
}

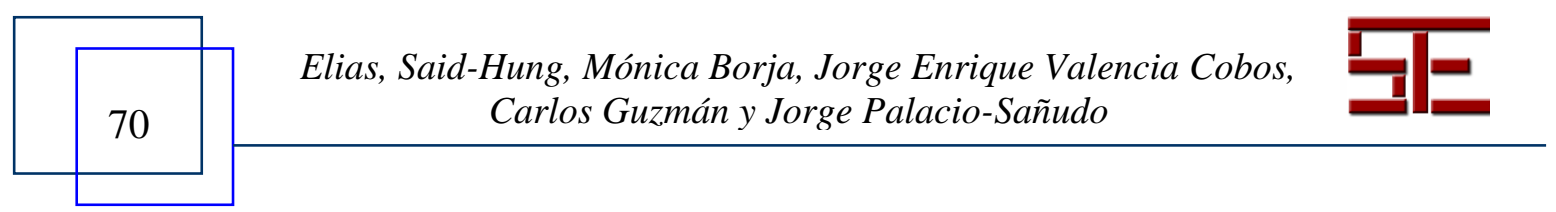




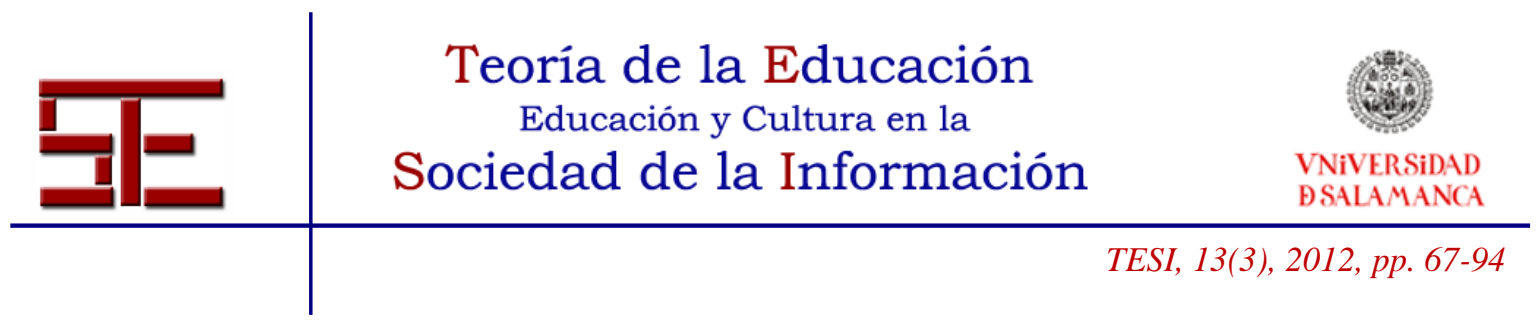

\section{2.- MARCO CONCEPTUAL}

\section{1.- Derecho a la educación y política educativa en Colombia}

La educación se constituye en posibilitador del reconocimiento y la concienciación del mundo, del sentido de la vida y de sí mismo, lo que se da en relación con el mundo, consigo mismo y con los otros (Charlot, 2008). De acuerdo con la Declaración Universal de los Derechos Humanos, en su artículo 26, la educación se plantea como un derecho que "se orientará al pleno desarrollo de la personalidad humana y al fortalecimiento del respeto a los derechos humanos y a las libertades fundamentales" (Naciones Unidas, 1948). Esta visión, ratificada por la CEPAL, OEI \& Secretaría General Iberoamericana (2010), no solo deja clara la importancia de la educación a nivel social, sino también, nos permite visualizar un panorama, donde se debe aún transitar un largo camino para garantizar el cumplimiento y la calidad de dicho derecho, en especial a nivel de Iberoamérica, y en Colombia, nuestro caso de estudio.

En las dos últimas décadas, Colombia (Banco Mundial, 2008) ha logrado avances importantes en cuanto a acceso a la educación y a la eficiencia interna del sistema educativo. No obstante, aún persiste el reto por la mejora de la calidad educativa, la cual se encuentra frente a un contexto crítico, si tomamos, por ejemplo, como referencia los resultados obtenidos por los estudiantes dentro del sistema educativo en las pruebas PISA de 2009 (ICFES, 2010); al no alcanzar el nivel 2 en dichas pruebas. Lo que equivale a decir que los estudiantes en Colombia, carecen de las habilidades básicas de lectura, lo que limitaría su participación, de manera proactiva y productiva, en la sociedad moderna. Este hecho hace que uno de los principales retos que en Colombia, sea avanzar en la promoción de un contexto donde se garantice no solo el acceso a los escenarios de enseñanza de los niños, niñas y jóvenes; sino también se garanticen la educación como un derecho efectivo para la mejora de las condiciones sociales en el país, y sea realizable, posible, un contexto social donde existan las condiciones favorables para el desarrollo y el progreso personal, social y económico de cada uno de los ciudadanos (Sarmiento, 2010).

Existen varios aspectos que deben ser tenidos en consideración, al momento de hacer un análisis exhaustivo en torno a las percepciones ciudadanas sobre el estado de la educación en Colombia. Dos de estos elementos son: la perspectiva del derecho de la educación, y el concepto de calidad educativa. Ambos, conceptos, más que reflejar un

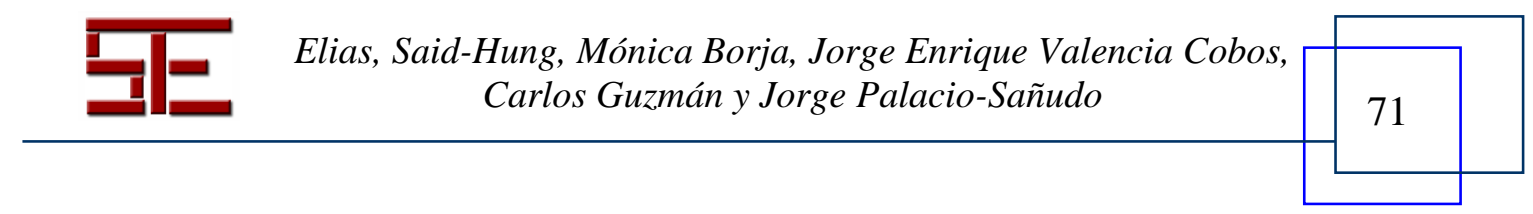




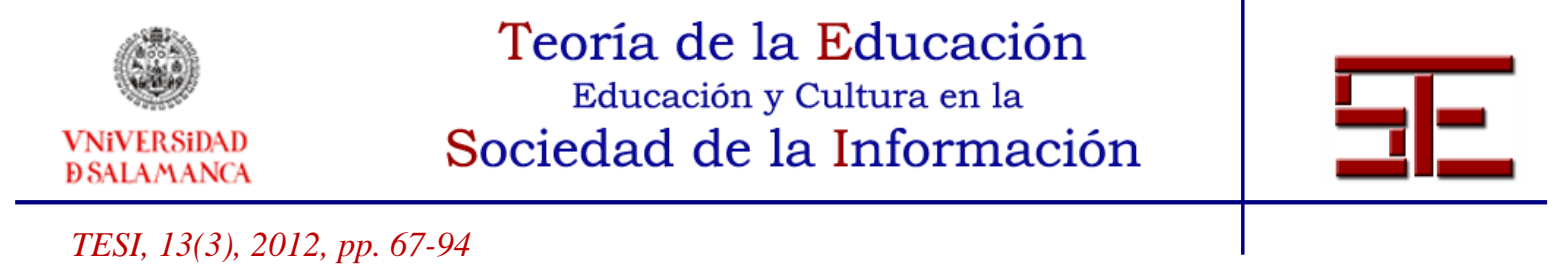

simple significado, nos permiten tener una aproximación más realista de la visión y acciones llevadas a cabo al interior de este sector, desde este país.

Como expone Lerma (2007), Colombia ha transitado desde comienzos de 1990 por distintas visiones que dan cuenta de la perspectiva de abordaje de la Educación en dicho país. De la aproximación de la educación como mecanismo de acumulación de capital humano y mejora del contexto productivo (económico) nacional, durante la primera mitad de la década de 1990; se ha pasado, desde la década de 2000, a concebir la educación como un factor de desarrollo humano, social y económico, a favor de la equidad social. A pesar de este avance conceptual general, los diferentes planes de desarrollo propuestos por los últimos gobiernos en Colombia, liderados por Andrés Pastrana (Departamento de Planeación Nacional, 2003), Álvaro Uribe (Departamento de Planeación Nacional, 2007), y el actual presidente, Juan Manuel Santos (Departamento de Planeación Nacional, 2011), no han sido reflejo de eficiencia pública que contribuyese a la promoción de un marco de garantía del derecho, fundamental, a la educación; sino que se centraron en aspectos vinculados con el acceso, la cobertura, la pertinencia y la eficacia en el sector (Tomasevski, 2004), vinculadas al fortalecimiento de competencias para el mejoramiento de la formación de capital humano, que ayudasen a la inserción productiva de los ciudadanos, entre otros aspectos.

\section{2.- La Calidad de la Educación.}

En medio del proceso político vinculado con el sector educativo en Colombia, la Calidad de la Educación ha sido objeto de un uso cada vez más recurrente por parte de los actores públicos y privados para aludir a tan importante área social. Pero, ¿cómo podemos definir la Calidad de la Educación? ¿Cómo ha sido empleado este concepto? ¿Cómo debemos concebir este concepto?

Como exponen Aziz, Canales et al. (2001), Marquès (2002), Toral (2003) y Vega (2008), entre otros, al momento establecer una aproximación conceptual en torno a la Calidad Educativa o en la Educación, resultan diversas las perspectivas en que han venido siendo consideradas para el tratamiento de este concepto.

En 1984, en el seno de la OCDE se asumió a la Calidad de la Educación como aspecto prioritario dentro del desarrollo de la política pública en muchos países. No sólo se han venido estableciendo un conjunto de calidades alrededor del tema educativo, desde entidades gubernamentales, centradas en: la calidad del currículo, la calidad de la

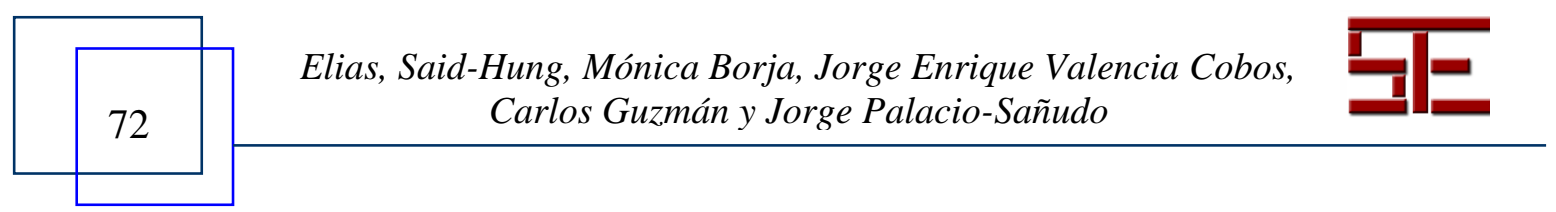




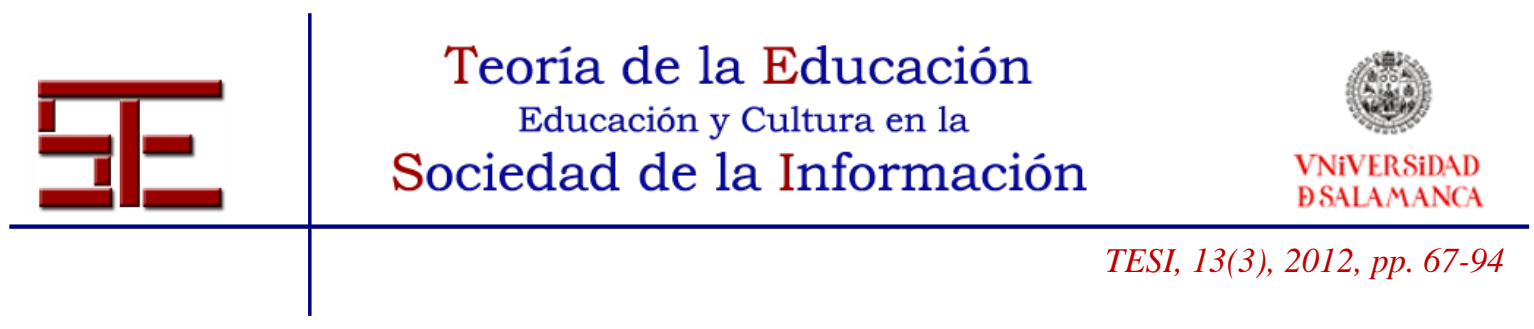

gestión escolar, la calidad de los docentes, la calidad de la evaluación y la calidad de la enseñanza; sino también, se han dado varias perspectivas que han contribuido al debate de este concepto.

Desde 1970 hasta nuestra fecha, a través de los trabajos publicados por Brookovel et al. (1979), Edmonds (1982), Purkey \& Smith (1983), Mortimore et al. (1998) y Creemers (1994), entre otros, se ha venido abordando el concepto de Calidad de la Educación o Educativa. En medio de esta búsqueda, surgen parámetros o intentos de demarcación desde distintas perspectivas: 1) como proceso que ayuda a garantizar a todos los jóvenes la adquisición de conocimientos, competencias y actitudes que les permitirán el tránsito a la vida adulta (OCDE, 1995); 2) como proceso, donde el sistema escolar les permite a los estudiantes sacar máximo provecho a las capacidades para la obtención de los mejores resultados o logros académicos, morales y emocionales (Mortimore et al., 1988); y 3) como un proceso, en el que el sistema educativo cuenta con la capacidad de ser accesible a todos los ciudadanos, de garantizar la participación activa de los alumnos en el aprendizaje y vida dentro de la institución educativa, así como la participación de las familias y resto de actores comunitarios alrededor de las escuelas, entre otros aspectos.

En todos los casos de aproximación conceptual del término Calidad de la Educación o Educativa, uno de los principales propósitos latente desde cada perspectiva es la búsqueda de parámetros medibles que ayuden a establecer una escala verificable que contribuya a la constitución de una pauta unificada de percepción en torno a este concepto. Es por ello que lo expuesto por Aron (1977) sigue estando vigente, al momento de tratar de armonizar aspectos cuantitativos y cualitativos que permitan la medición de tan importante concepto. Entre 1980 y 1990, surgen una variedad de aproximaciones a favor del diseño de un mecanismo de evaluación de la educación; en su mayoría de corte cuantitativo que propenden la medición de este sector que garanticen el diseño de políticas públicas efectivas, desde la puesta en marca de modelos de medición orientados, en su mayoría, a: 1) la rendición de los logros y rendimientos académicos a nivel educativo; 2) la explicación del logro o no de determinados objetivos educativos; 3) la evaluación en el cumplimiento de los marcos normativos que regulan el sistema educativo; y 4) el establecimiento de relaciones causales entre el contenido de determinadas políticas educativas y fenómenos educativos observados dentro de las instituciones educativas (Tiana \& Santángelo, 1994). Es así como, en la actualidad, disponemos, a nivel internacional, regional y nacional, de diferentes herramientas de medición alrededor de la calidad, como por

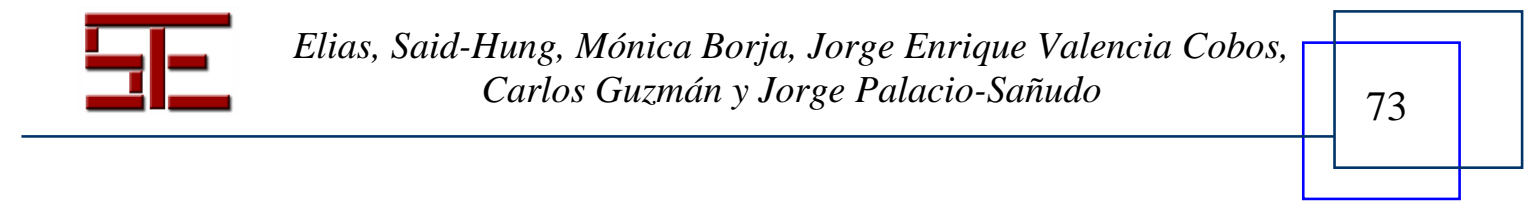




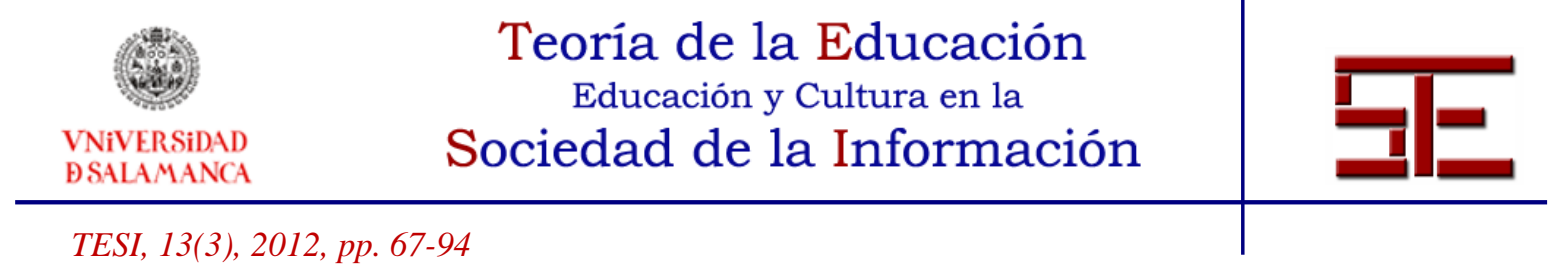

ejemplo: las Pruebas PISA ${ }^{2}$, TIMSS o PIRLS ${ }^{3}$; a nivel regional (Iberoamérica) se ha promovido el estudio $\mathrm{SERCE}^{4}$; y a nivel nacional, en nuestro caso en Colombia, las Pruebas $\mathrm{SABER}^{5}$; cada una de estas orientada más a la medición del rendimiento académico y competencias adquiridas por los alumnos.

A pesar de la diversidad de mediciones que se han venido planteando para el abordaje de parámetros claros que ayuden al establecimiento de la Calidad de la Educación, muchas veces estas propuestas no logran concretar un acercamiento asertivo de todos los factores que inciden directamente en este concepto.

Al momento de hablar de los factores determinantes en la Calidad Educativa, nos volvemos a encontrar con un contexto marcado por la diversidad de perspectivas. Investigadores como Vélez, Schiefelbein \& Valenzuela (1996), Aziz, Canales et al. (2001), Marquès (2002), Willms, Sommers, \& Pardo (2001), entre otros, han venido debatiendo sobre esta temática; llegando a identificar como factores asociados a este concepto aquellos vinculados con: el insumo desde las instituciones educativas (formación docente, formación del alumnado, dotación de equipos, infraestructura y materiales escolares); el contexto educativo, relacionado con la gestión y autonomía institucional; y aspectos relacionados con el contexto social donde se desenvuelve la vida académica de los estudiantes, los docentes y las instituciones educativas (la familia, ingreso familiar, carácter monoparental o no de los núcleos familiares, nivel cultural, entre otros). Cada uno de estos factores no ha gozado de tratamiento igualitario por parte de investigadores y entidades; sino, por el contrario, resulta su abordaje diverso, según la perspectiva de conceptualización desde donde se parte al momento de conceptualizar a la Calidad Educativa.

Con base a lo expuesto en este apartado, el acercamiento del concepto, desde una posición donde se propende a la conformación de una posición intermedia, frente a las diferentes percepciones expuestas al comienzo de este punto. Es decir, para nosotros, la Calidad Educativa, si bien debe ser entendida como un proceso que ayuda a los estudiantes a la adquisición de conocimientos, competencias y actitudes para afrontar la

\footnotetext{
${ }^{2}$ http://www.oecd.org/.

${ }^{3}$ http://timss.bc.edu/.

${ }^{4}$ http://hydra.icfes.gov.co/serce/.

${ }^{5}$ http://www.icfes.gov.co/saber59/.
}

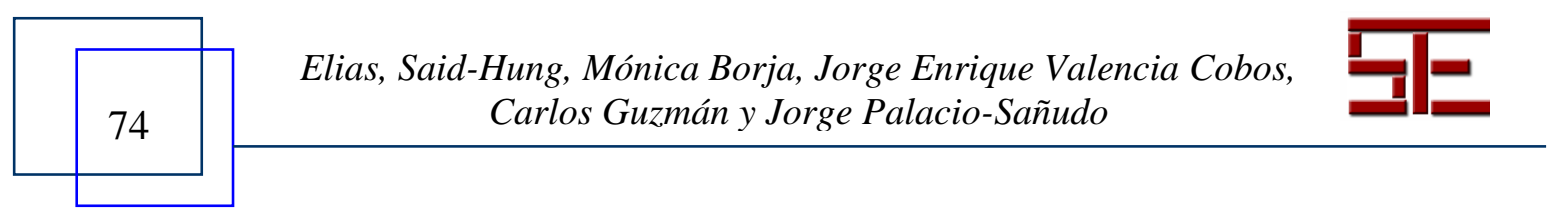




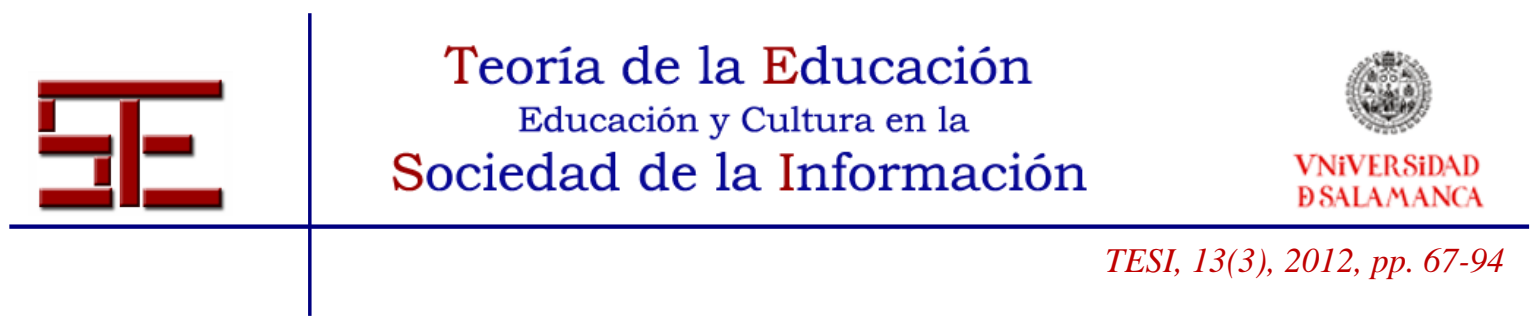

vida adulta, el cual no se logrará sin garantizar que: 1) los estudiantes adquieran capacidades cognitivas, emocionales y moralizantes que les permitan ser unos individuos socialmente exitosos; y 2) se concrete la participación activa de los actores educativos y sociales, para la concreción del fin expuesto al comienzo.

2.3.- Percepción ciudadana de la educación

La percepción ciudadana implica experiencias que se reciben por los sentidos, pero también una idea que se construye por la información que acumulamos sobre situaciones que nos afectan directa o indirectamente. La educación, por otro lado, puede ser pensada como una mera transmisión de conocimientos y prácticas "científicas", hasta la construcción de sociedades democráticas. Cada sociedad y cada individuo hacen una selección de acuerdo con el "ethos de clase" de Bourdieu (1973), es decir, un sistema de valores que las personas han interiorizado desde la infancia, los cuales son empleados para la generación de respuestas a problemas distintos (Bourdieu, 2000).

Al hablar sobre la percepción que tienen los ciudadanos en torno a la educación, Brunner (2000) manifiesta que dicha percepción varía según los grupos de ciudadanos: para el sector dirigente, los medios de comunicación y los organismos internacionales, la educación ocupa un papel central en los discursos relacionados con el desarrollo de una nación; mientras que, para los ciudadanos y las asociaciones civiles, la educación es vista como un bien o recurso que posibilita la movilidad social. Es desde esta diferencia de perspectivas que, desde este artículo, se plantea la necesidad de ahondar mecanismos que ayuden a la comprensión en torno a la educación desde los diferentes actores sociales, en especial, desde los ciudadanos, ya que serán estos quienes estarán a cargo de ejercer presión, a través de la movilidad social expuesta, a los actores públicos y privados involucrados con el proceso de mejora o no del sector educativo existente en cada país, en nuestro caso en Colombia.

Como bien lo señala Gil-Pérez y Vilches (2005), aún resulta bastante común la falta de mecanismos sociales que garanticen la participación más directa de todos los actores involucrados en la formulación, aplicación y resultados generados desde el proceso de diseño de las políticas y planes públicos orientados al mejoramiento de la educación, en cada país. Es por lo antes expuesto que la aplicación de encuestas orientadas a la percepción ciudadana para el delineo de políticas y planes puede resultar un mecanismo por demás interesante, al momento de: 1) orientar la toma de decisiones aplicadas en el sector educativo (Lora, 2008); 2) al momento de establecer fluctuaciones y expectativas relacionadas con los niveles de satisfacción que tienen los ciudadanos, en general, en

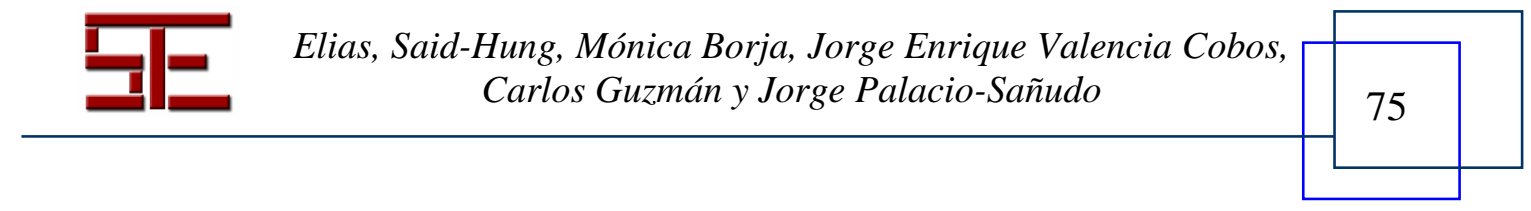




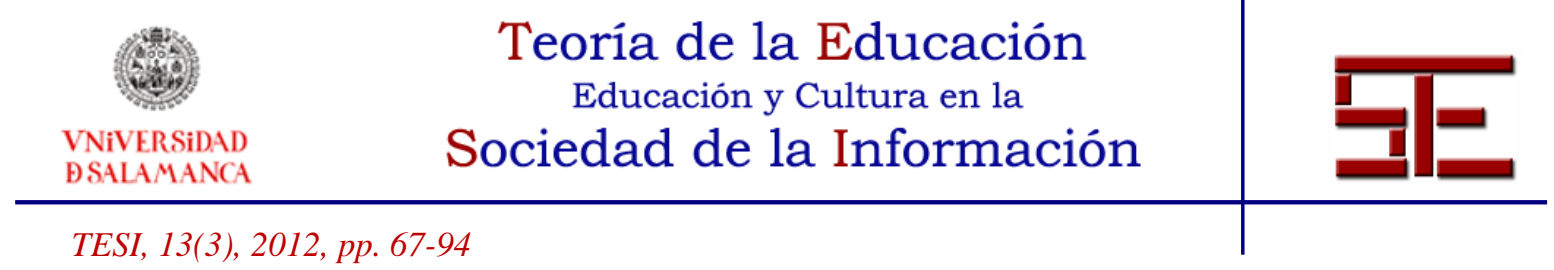

torno al estado y acciones aplicados a nivel educativo; y 3) a una mayor comprensión de las diferentes conciencias relacionadas con las deficiencias presentadas alrededor de la Calidad Educativa, desde el significado y comprensión que tienen los ciudadanos sobre este concepto y de los diferentes procesos que guardan relación con ella.

Estudios realizados por Elacqua y Fábrega (2006), Mizala y Urquiola (2007) o Vegas y Petrow (2007), por citar algunos, han dado cuenta de la valía de los estudios percepción ciudadana, en especial a nivel de Iberoamérica, para los fines expuestos en el párrafo anterior; en especial al momento de identificar el mayor número de detalles que puedan ayudar al delineo de políticas públicas eficaces (Lora, 2008). Ello, desde la puesta en marcha de instrumentos que ayuden no solo al fortalecimiento de las fuentes de información cuantitativas que hoy se han venido fortaleciendo a nivel internacional y nacional, para la medición de la Calidad Educativa; sino también a la identificación de las brechas que puedan estar existiendo entre lo que el ciudadano percibe y la realidad educativa en cada país, región o en el mundo.

\section{3.- METODOLOGÍA}

La encuesta, en la que se basa este análisis expuesto en este artículo, fue realizada a hombres y mujeres, mayores de 18 años de edad, de todos los niveles socio económicos (estratos), residentes habituales en el área urbana de las ciudades de Bogotá, Medellín, Cali, Manizales, Cartagena, Quibdó y Soacha, en Colombia. La muestra de tipo estratificado, polietápico, estuvo compuesta por 1.640 encuestas, ponderadas por ciudad, nivel socioeconómico, género y rangos de edad, con un nivel de confianza del $95 \%$ y un error muestral de $+/-2.6 \%$.

Para la recolección de datos, la Encuesta Nacional de Percepción Ciudadana sobre Educación ${ }^{6}$ dio cuenta de la técnica de entrevista personal en hogares, con aplicación de un cuestionario estructurado. Los datos procesados y validados de la encuesta se reagruparon en tres grupos de variables, basándose en la revisión teórica y la

\footnotetext{
${ }^{6}$ Encuesta realizada por la empresa IPSOS Napoleón Franco (http://www.ipsos.com.co/), quien estuvo a cargo del levantamiento de la encuesta financiada por Educación Compromiso de Todos marzo de 2010. El trabajo realizado por la empresa a cargo de la realización de este estudio tomó para la selección de las unidades muestrales el último censo de población y vivienda de 2005 realizados por el DANE, con sus correspondientes proyecciones al 2010.
}

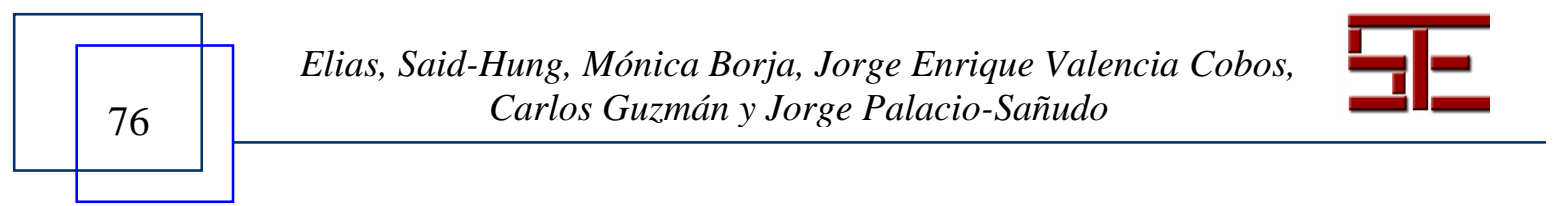




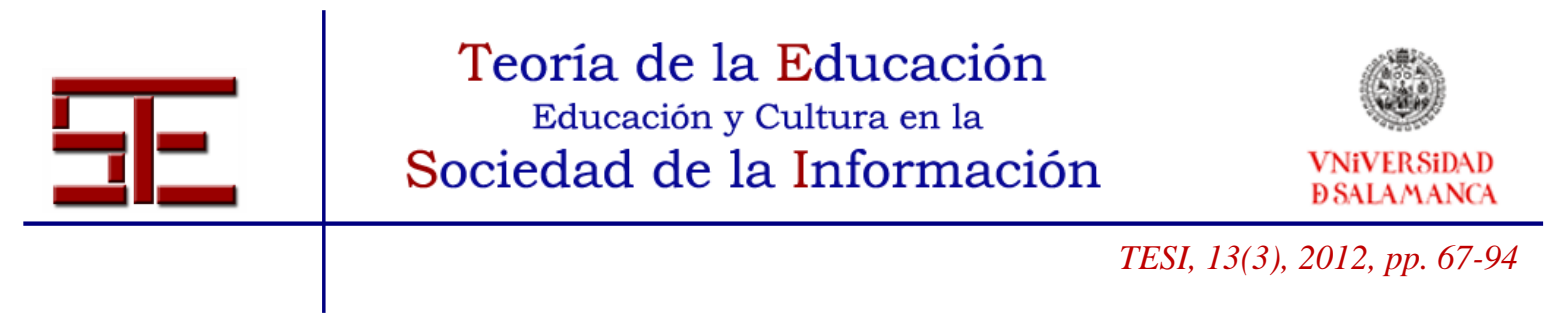

experiencia previa de los investigadores responsables del desarrollo del proyecto que dio origen a este artículo: variables socio demográficas, variables asociadas a la percepción y conocimiento del derecho a la educación, y variables asociadas a la percepción de los procesos de calidad educativa.

Una vez reagrupados los datos, se determinó la consistencia interna de las unidades de medición, a través de la relación presentada entre los elementos que lo conforman; a partir de la aplicación de una prueba de falibilidad a cada grupo de variables dentro de cada categoría tomada para el desarrollo de este artículo; evaluándose así la consistencia de cada escala creada para la encuesta en mención, omitiendo del análisis aquellas variables sin relación directa con el sistema educativo, así como aquellas preguntas abiertas cuya clasificación se presumió arbitraria. Al realizar la reorganización de las variables y verificada la consistencia en la creación de las escalas, se evaluó el nivel de asociación de cada una de las variables y categorías obtenidas con una variable dependiente que refleja la satisfacción del entrevistado con el sistema educativo: ¿Qué tan insatisfecho o satisfecho se siente usted en general con la educación que están recibiendo los niños y jóvenes?, la cual fue recodificada en una variable dicotómica que da cuenta de la satisfacción o insatisfacción de cada entrevistado con el sistema educativo nacional. Para este proceso, se tomaron en cuenta el cálculo de frecuencias, medias y distribuciones porcentuales; así como el test $\mathrm{JI}^{2}$ y los coeficientes Phi y V de Cramer, para variables categóricas, y los estadísticos Tau-b, Tau-c de Kendall, para variables ordinales. El análisis inferencial consistió en la aplicación de un modelo de regresión logística que diese cuenta de la probabilidad de que un ciudadano en Colombia esté satisfecho o no.

\section{4.- RESULTADOS EN TORNO A LA PERCEPCIÓN CIUDADANA DE LA SITUACIÓN EDUCATIVA EN COLOMBIA}

Tal como se aprecia en la Tabla 2, el nivel de satisfacción en el sistema escolar en Colombia fue más favorable, como resultado del $\mathrm{P}$-valor $\leq 0,05$ de las variables independientes consideradas para este estudio, cuando los encuestados hacían parte de un estrato socio-económico más alto; eran mujeres con mayor edad y con un mayor nivel de formación; y de acuerdo con la ciudad donde habitaban en dicho país.

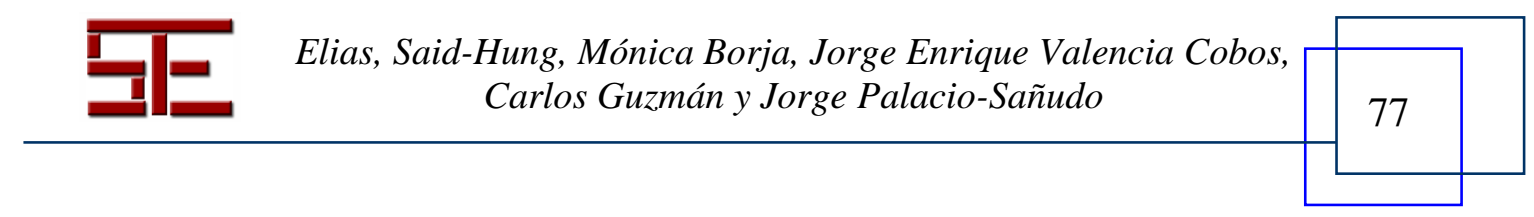




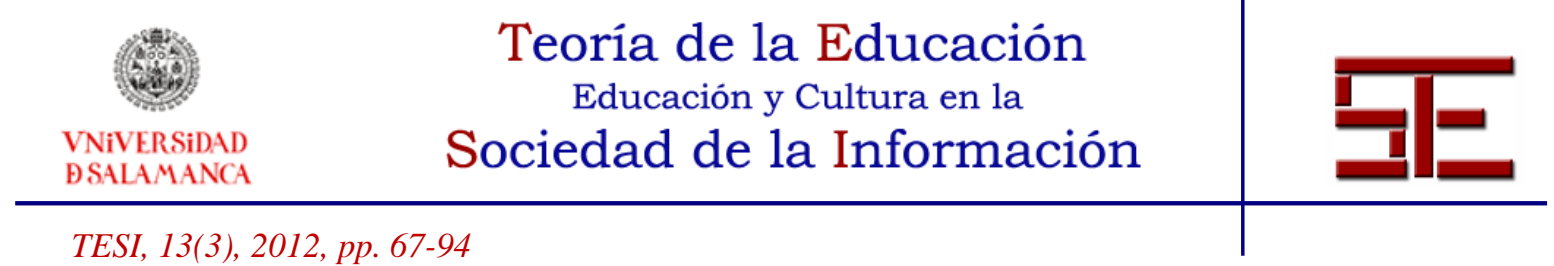

Una de las variables independientes que inciden significativamente en torno al nivel de satisfacción que se tiene en Colombia del sistema educativo es la ciudad donde se habita en dicho país. A pesar de no poder establecerse una posible relación entre el PIB departamental por habitante y el nivel de satisfacción del sistema educativo por ciudad, la Figura 1 y la Tabla 1, nos permiten ver cómo, en general, el nivel de satisfacción promedio (siendo 5 el valor máximo de valoración emitido por los encuestados) con el sistema educativo de la mayoría de las ciudades es muy cercano al promedio nacional, sin embargo, en la ciudad de Cartagena de Indias (Colombia), la media del nivel de satisfacción entre los encuestados resultó estar más próxima a la opción "más favorable", a pesar de que dicha ciudad presenta unos de los niveles de PIB por habitante más bajos del conjunto de ciudades tomadas para el desarrollo de la encuesta en la que se basa este artículo. En contraposición, las ciudades con mayores niveles de PIB por habitante (Bogotá, Cali y Medellín) presentan niveles de satisfacción más cercanos al promedio nacional.

Figura 1: PIB departamental por habitante y promedio del nivel de satisfacción por ciudad

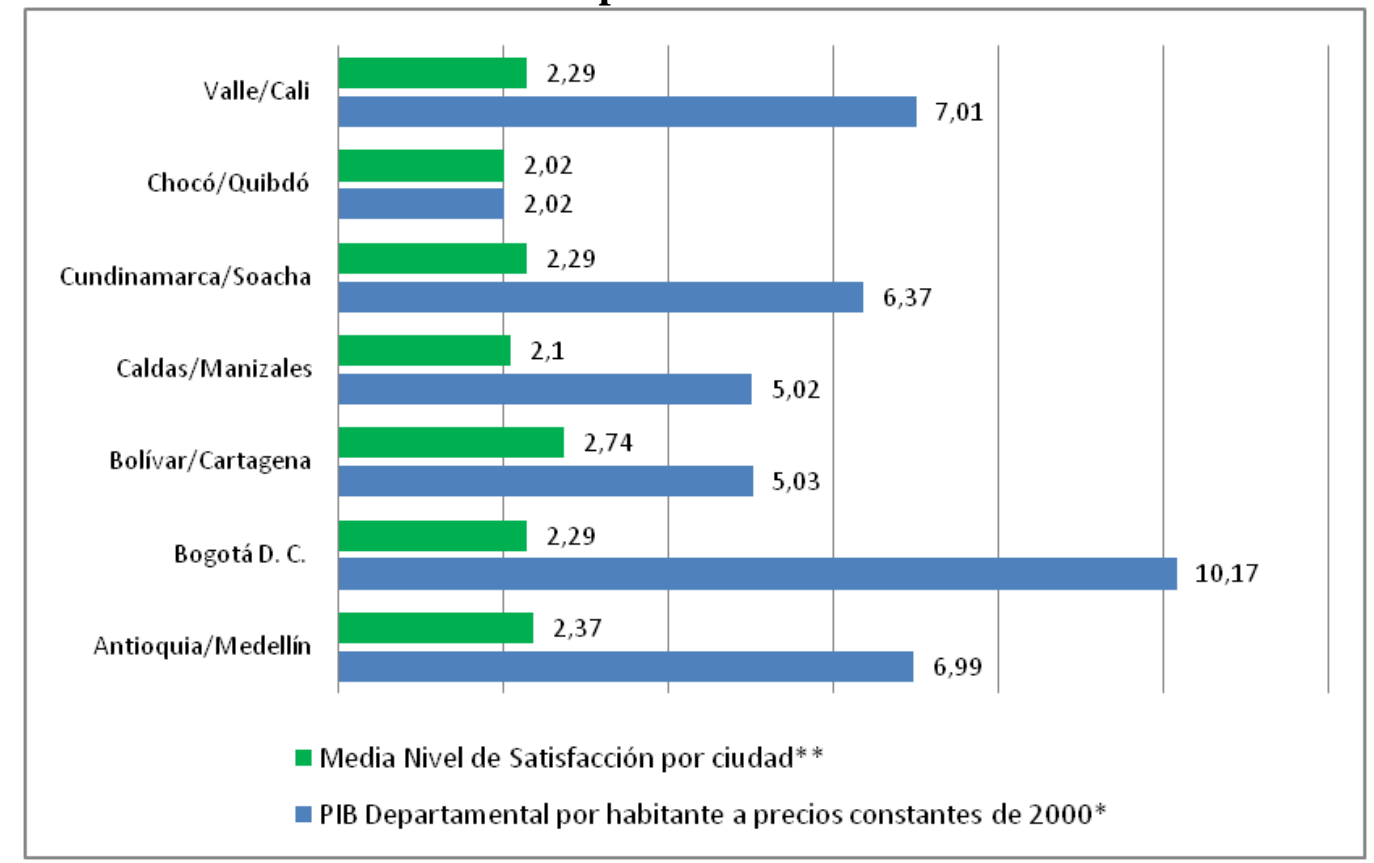

Fuente: DANE - Cuentas regionales año 2007

Dato: *Cifras en millones de pesos

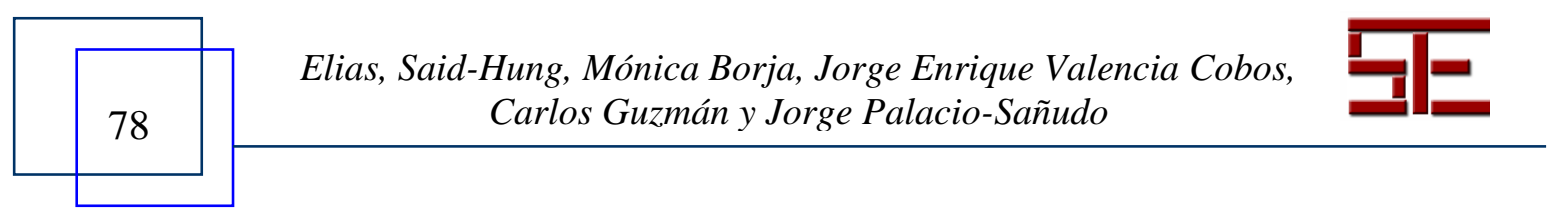




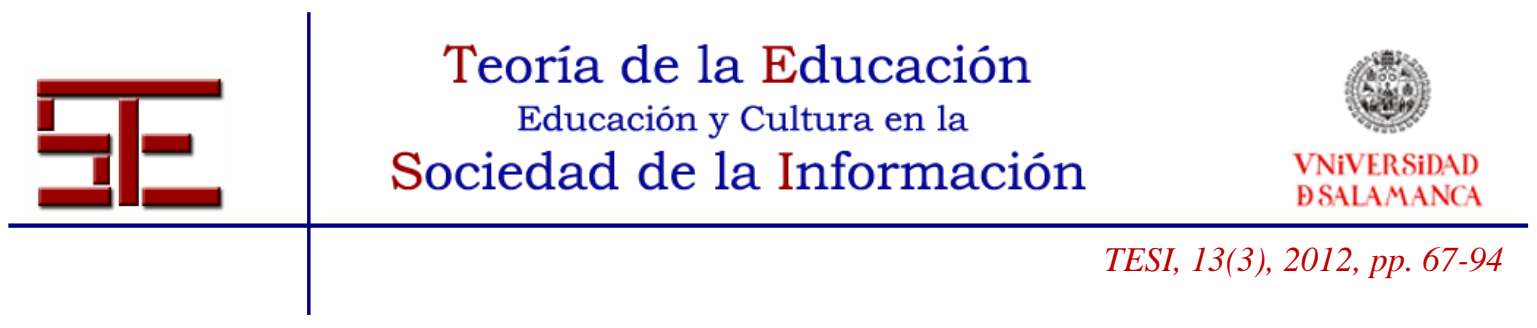

** 1=Nivel de satisfacción bajo; 2=Nivel de satisfacción medio; 3=Nivel de satisfacción alto.

Tabla 1: Relación entre el nivel de satisfacción del sistema escolar en Colombia y variables socioeconómicas

\begin{tabular}{|c|c|c|c|c|c|c|c|c|}
\hline \multirow{2}{*}{$\begin{array}{c}\text { Variables } \\
\text { Socioeconómicas }\end{array}$} & \multicolumn{5}{|c|}{ Estadísticos descriptivos } & \multicolumn{3}{|c|}{$\begin{array}{l}\text { Pruebas de Chi- } \\
\text { cuadrado }\end{array}$} \\
\hline & $\mathrm{N}$ & Mínimo & Máximo & Media & $\begin{array}{l}\text { Desv. } \\
\text { típ. }\end{array}$ & Valor & $\mathrm{gl}$ & $\begin{array}{c}\mathrm{p}- \\
\text { valor }\end{array}$ \\
\hline Estrato* & 1640 & 1 & 6 & 2,47 & 1,241 & 39,314 & 10 &, 000 \\
\hline Ciudad** & & 1 & 7 & 3,54 & 2,130 & 146,899 & 12 &, 000 \\
\hline $\operatorname{Edad}^{* * *}$ & & 1 & 6 & 3,06 & 1,583 & 18,519 & 10 & ,047 \\
\hline Genero $* * * *$ & & 1 & 2 & 1,50 &, 500 & 12,968 & 2 & ,002 \\
\hline Nivel Educativo $^{\wedge}$ & & 1 & 13 & 6,28 & 2,233 & 61,594 & 24 &, 000 \\
\hline
\end{tabular}

Fuente: Elaborada por los autores.

Datos: Variable Dependiente Nivel de Satisfacción con el Sistema Escolar

* Estratos 1, 2, 3, 4, 5, 6 .

** 1 = Bogotá, 2 = Medellín, 3 = Manizales, 4 = Cali, 5 = Quibdó, $6=$ Cartagena, 7 =

Soacha.

*** 1 = De 18 a 24 años, $2=$ De 25 a 34 años, $3=$ De 35 a 44 años, $4=$ De 45 a 54 años, 5

$=$ De 55 a 64 años, $6=$ Más de 64 años.

$* * * * 1=$ femenino, $2=$ masculino

^ 1 = Sin estudios, 2 = Sin estudios, pero sabe leer y escribir, 3 = Primaria incompleta, $4=$ Primaria completa, $5=$ Secundaria incompleta, $6=$ Secundaria completa, $7=$ Técnico $/$ tecnólogo incompleto, $8=$ Técnico/ tecnólogo completo, $9=$ Universitario $=$ Doctorado. incompleto, 10 = Universitario completo, $11=\quad$ Especialización, $12=$ Maestría, 13

Los niveles de asociación observados entre los factores educativos y sociodemográficos $(\mathrm{P}$-valor $\leq 0.05)$ y los niveles de satisfacción del Sistema Educativo en Colombia entre los encuestados, nos lleva a preguntarnos ¿Qué otros factores pueden estar generando este comportamiento? Si bien el cruce de datos expuesto en la Tabla 1 solo nos permite hacer comparaciones de aspectos encontrados a nivel social entre los encuestados del proyecto aquí tratado, los resultados extraídos de la encuesta nos permiten indicar la no relación del tamaño de estas ciudades, respecto al nivel de satisfacción analizado (Tabla 2). Por tanto, la no presencia de asociación orienta hacia un comportamiento homogéneo en la percepción de satisfacción con el sistema educativo, más relacionado con aspectos de índole socio-culturales, política y económica que con la ubicación geográfica.

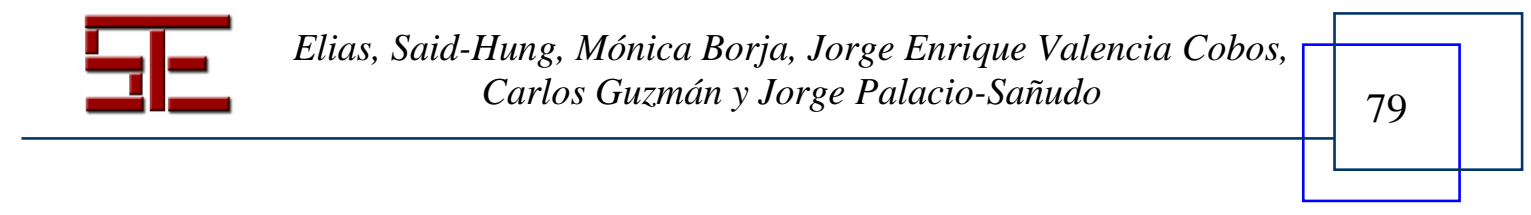




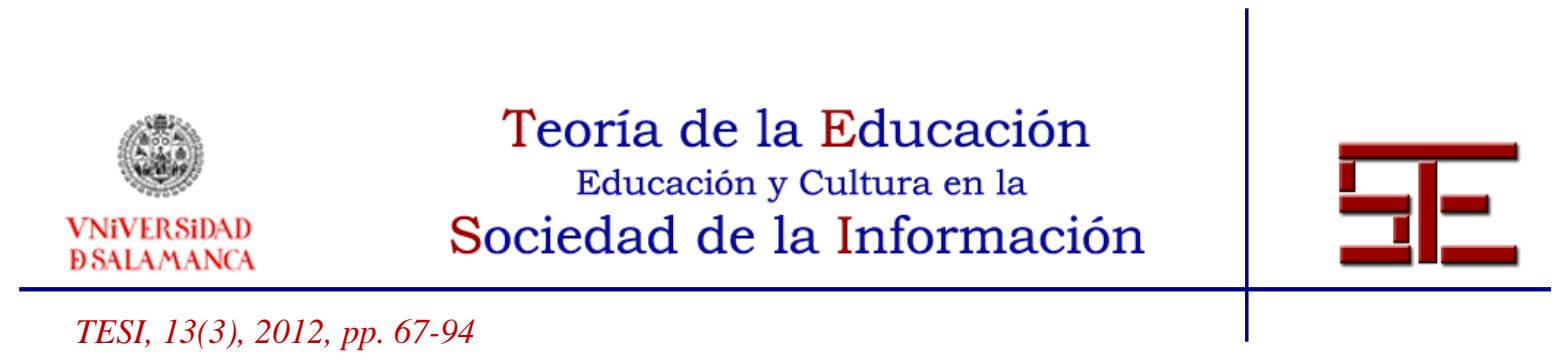

Tabla 2: Pruebas de chi-cuadrado del nivel de satisfacción del sistema educativo en Colombia, según el tamaño de la ciudad

\begin{tabular}{lcrr}
\hline & Valor & gl & $\begin{array}{c}\text { Sig. Asintótica } \\
\text { (bilateral) }\end{array}$ \\
\hline Chi-cuadrado de Pearson &, 620 & 2 &, 734 \\
Razón de verosimilitudes &, 623 & 2 &, 732 \\
Asociación lineal por lineal &, 619 & 1 &, 431 \\
N de casos válidos & 1640 & & \\
\hline
\end{tabular}

Fuente: Elaborada por los autores.

Datos: $a=0$ casillas $(, 0 \%)$ tienen una frecuencia esperada inferior a 5 . La frecuencia mínima esperada es 71,20 .

Por último, debe destacarse la no presencia de relación estadísticamente significativa entre la edad y la satisfacción con el sistema escolar (p-valor 0,225). Esta particularidad ha sido destacada en análisis previos, como por ejemplo los expuestos por Lora (2008), donde, a través de los estimadores ${ }^{7}$, la variable edad no fue diferente de cero con significancia estadística.

\section{1.- Variables asociadas a la percepción del derecho a la educación en Colombia}

En lo que se refiere a las variables asociadas a la percepción y conocimiento del derecho a la educación, los datos en los que se basa este artículo nos permiten ver la relación estadísticamente significativa que hay entre los niveles de satisfacción del sistema educativo en Colombia y la percepción que mostraron los encuestados en torno a la cantidad que se invierte en materia educativa que se está haciendo a nivel local, en cada una de las ciudades donde residían; así como también la percepción que tenía cada encuestado sobre los niveles de garantía al derecho a la educación que reciben actualmente esto, tanto a nivel personal como familiar (Tabla 3). Es decir, el nivel de satisfacción del sistema educativo en Colombia fue más favorable entre los encuestados que estimaron que "sus ciudades", regidas por actores públicos como Gobernaciones, Alcaldías y Secretarías de Educación, mostraban una capacidad de inversión o prioridad dirigida a la atención de la educación a nivel local. Estos niveles de satisfacción fueron más favorables entre los encuestados cuando estos reconocían que, tanto a nivel personal como familiar, sus derechos a la educación eran garantizados.

\footnotetext{
${ }^{7}$ Obtenidos de un modelo de Regresión Logística Binaria, con el cual se pretende expresar la probabilidad de que ocurra el evento en cuestión como función de ciertas variables, que se presumen relevantes o influyentes sobre le comportamiento de una variable categóricas que sólo puede tomar dos valores
}

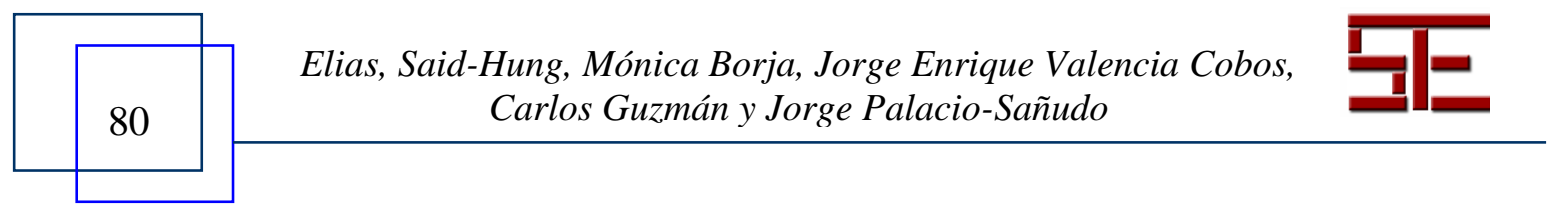




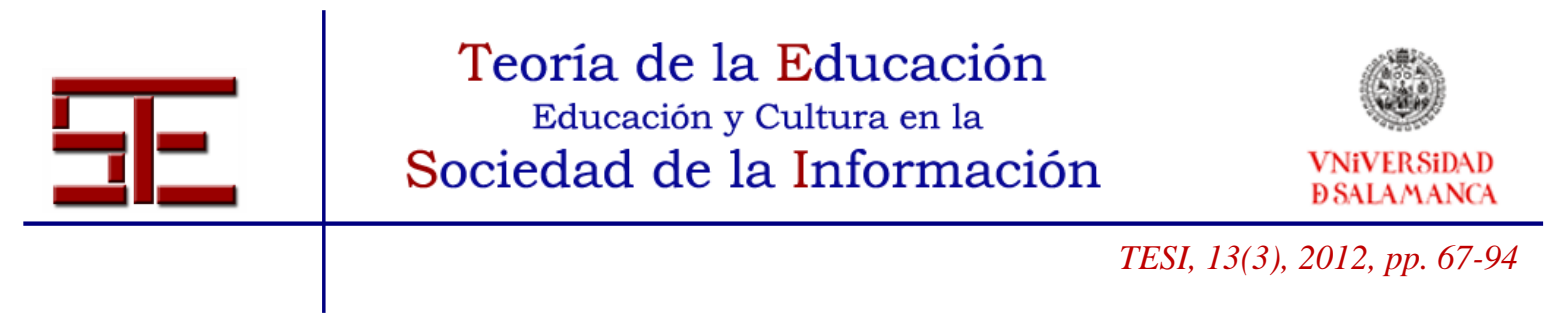

La alta satisfacción que presentan los colombianos frente a la educación coincide con lo expuesto por Lora (2008), donde se anota que la opinión pública manifiesta una aceptación respecto de la calidad educativa en la región.

Tabla 3: Relación entre los niveles de satisfacción sobre sistema educativo en Colombia y la capacidad de inversión local en materia educativa y la garantía del derecho a la educación

\begin{tabular}{|c|c|c|c|c|c|c|c|c|}
\hline \multirow{2}{*}{$\begin{array}{c}\text { Variable Categoría Derecho a la } \\
\text { Educación }\end{array}$} & \multicolumn{5}{|c|}{ Estadísticos descriptivos } & \multicolumn{3}{|c|}{$\begin{array}{l}\text { Pruebas de chi- } \\
\text { cuadrado** }\end{array}$} \\
\hline & $\mathrm{N}$ & Mínimo & Máximo & Media & típ. & Valor & $\mathrm{gl}$ & p-valor \\
\hline $\begin{array}{l}\text { - USted cree que su ciudad está } \\
\text { invirtiendo lo que se requiere para el } \\
\text { tema de educación? } *\end{array}$ & 1640 & 0 & 1 &, 25 &, 433 & 114,288 & 2 &, 000 \\
\hline 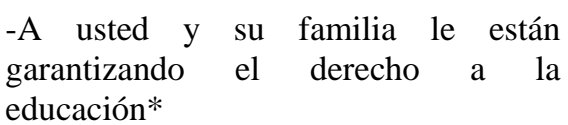 & 1539 & 0 & 1 & ,67 & ,469 & 103,326 & 2 &, 000 \\
\hline
\end{tabular}

Fuente: Elaborada por los autores.

Dato: $* 0=\mathrm{No} ; 1=\mathrm{Si}$

**Variable Dependiente Nivel de Satisfacción con el Sistema Escolar.

\section{2.- Variables asociadas a la percepción de los procesos de calidad educativa en Colombia}

El último subgrupo de preguntas identificadas como variables asociadas a la percepción de los procesos de calidad educativa en Colombia, clasificadas en: formación docente, gestión escolar, infraestructura, acceso y metodologías, nos permiten ver como algunos factores intra-escolares resultan de mayor interés, al momento de evaluar como favorable la situación del sistema educativo colombiano. Los resultados expuestos en la Tabla 4 nos permiten ver cómo el nivel de satisfacción fue más favorable, en el caso de la formación docente, cuando los encuestados manifestaron tener una mejor percepción en torno a los niveles de preparación de los profesores, la actualización formativa de estos y el nivel de profesionalización en ellos.

Este aspecto es identificado por Arancibia (1994), Castilla (2005) y Marinho (2007), entre otros; quienes reconocen la formación docente como un factor esencial en la consecución de procesos de enseñanza eficaces y de los procesos de reforma curriculares que propendan por la Calidad de la Educación.

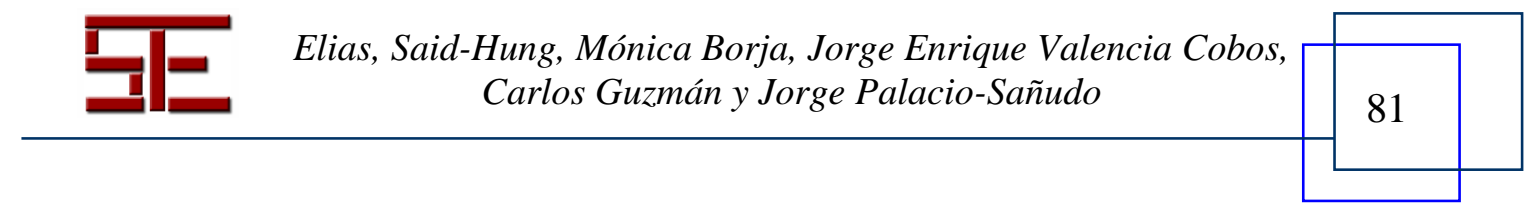


Tabla 4: Relación entre el nivel de satisfacción del sistema educativo en Colombia y la formación docente, gestión escolar, infraestructura, acceso y metodologías

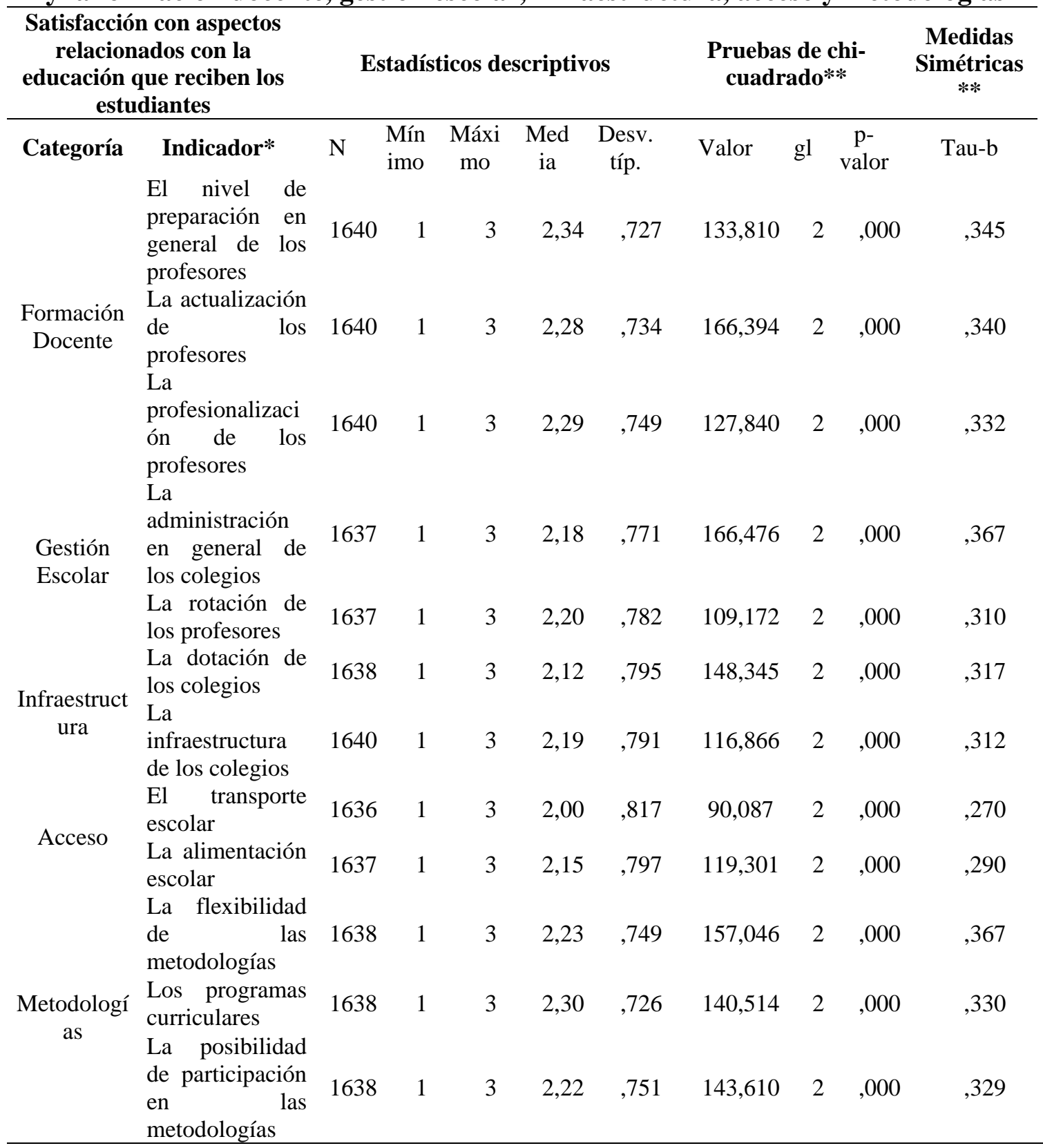

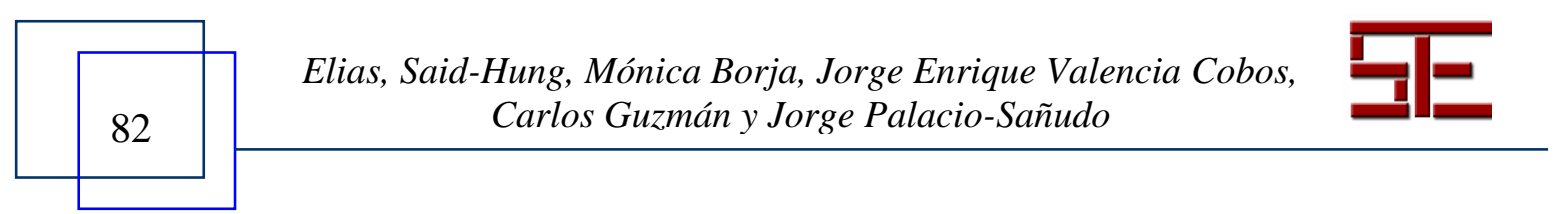




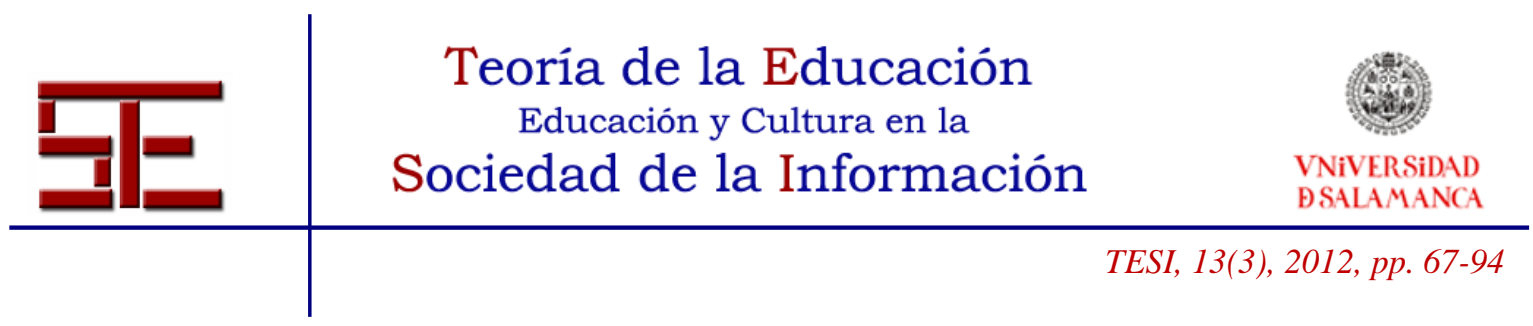

Fuente: Elaborada por los autores.

Dato: $*$ Bajo $=1$; Medio $=2$; Alto=3

**Variable Dependiente Nivel de Satisfacción con el Sistema Escolar.

En lo que se refiere a la gestión escolar (Tabla 4), las variables independientes relacionadas con el nivel de satisfacción del sistema escolar en Colombia fueron aquellas que aludían a cómo se administraban las instituciones educativas, en general, y las que hacían referencia a la rotación de los profesores dentro de estos escenarios de enseñanza. Es decir, los niveles de satisfacción fueron más favorables cuando la percepción de los encuestados, en torno a los aspectos aquí expuestos, era alta. Lo anterior guarda coherencia con los estudios realizados por Herrera \& López (1996), Rodríguez (2000) y la CEPAL \& UNESCO (2005), quienes señalan que los aspectos relacionados con este factor resultan ser críticos para las escuelas eficaces; y con la visible relación existente entre la gestión administrativa, los procesos de enseñanza, niveles de escolaridad de la madre y los resultados de los aprendizajes.

En cuando a la infraestructura, las variables independientes con relación significativa $(\mathrm{P}$-valor $\leq 0.05)$ con los niveles de satisfacción del sistema escolar en Colombia fueron dos: la dotación de las instituciones educativas y la infraestructura en estos escenarios. Es decir, los encuestados tuvieron un nivel de satisfacción más favorable del sistema educativo cuando calificaron como alta la percepción de tenían en torno a lo antes expuesto.

Las variables independientes vinculadas con el acceso al sistema educativo que presentaron una relación significativa $(\mathrm{P}$-valor $\leq 0.05)$ con el nivel de satisfacción del sistema educativo en Colombia entre los encuestados fueron aquellas relacionadas con el transporte y la alimentación escolar; es decir, los niveles de satisfacción aquí tratados fueron más favorables en aquellos encuestados cuya percepción fue alta en cuanto a la satisfacción que tenían alrededor del transporte y la alimentación prestada en las instituciones educativas.

Actualmente, las consideraciones, en cuanto a elevar la calidad de la educación, han permitido mejorar los indicadores de cobertura; llegándose a afirmar en torno a esta región que: "Durante la década pasada, el porcentaje de niños que ingresaron y terminaron la educación primaria y secundaria aumentó más rápidamente en América Latina que en cualquier otra parte del mundo en desarrollo" (PREAL, 2005:10). No obstante, aún hace falta mucho por hacer sobre los avances alrededor de los Objetivos

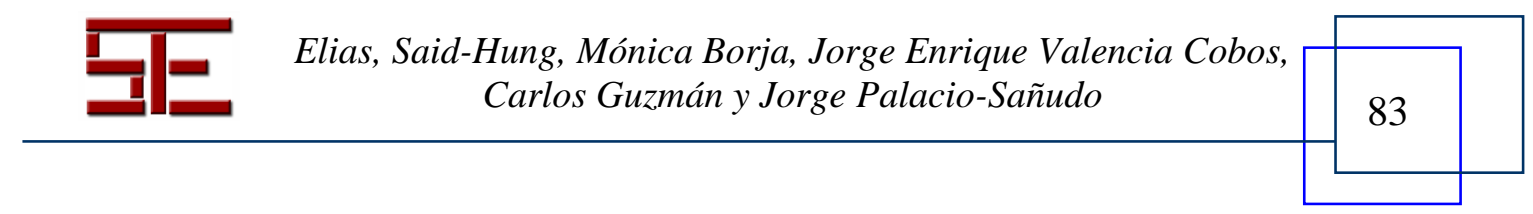




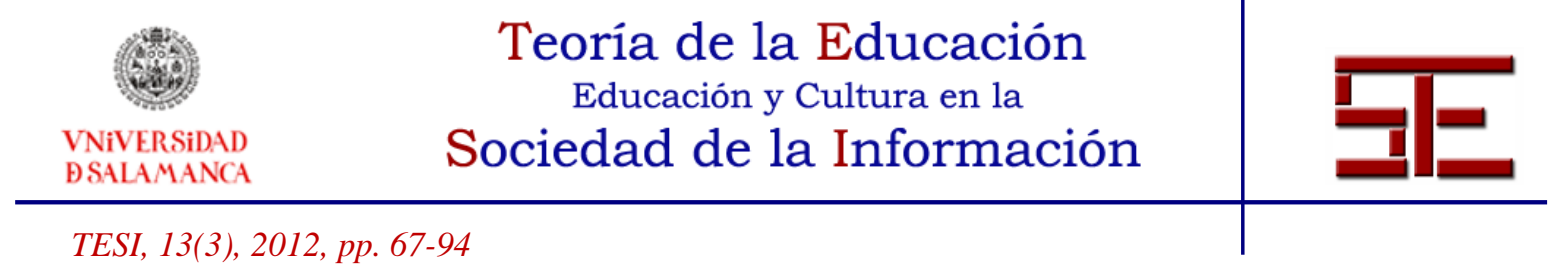

de Desarrollo del Milenio (Naciones Unidas, 2010). Tal como lo expone el Ministerio de Educación Nacional de Colombia (2007), la inversión en educación debe ser una prioridad en los países latinoamericanos, en nuestro caso en Colombia, si lo que se quiere es que ésta se convierta en un vehículo para mejorar el desarrollo económico y social de la población. No obstante, hay que señalar que "los esfuerzos no solo se deben centrar en el aumento de la oferta educativa sino también en el aseguramiento de las condiciones que fomenten el acceso efectivo a estos servicios, lo que involucra frecuentemente intervenciones de carácter multisectorial" (OEI, 2010:39).

El último grupo de variables independientes con relación significativa $(\mathrm{P}$-valor $\leq 0,05)$ con el nivel de satisfacción del sistema escolar en Colombia, por parte de los encuestados, guardan relación con las metodologías aplicadas al interior de las aulas e instituciones educativas, a saber: la flexibilidad de las metodologías, los programas curriculares existentes en la actualidad y las posibilidades de participación brindadas desde las metodologías aplicadas en el aula de clase. Por tanto, el nivel de satisfacción del Sistema Escolar en Colombia fue más favorable en aquellos encuestados cuya percepción daba cuenta de una alta satisfacción en torno a los temas abordados en las variables aquí mencionadas.

El diseño, la gestión y la evaluación de los procesos formativos que realiza el docente llevan implícita una carga de creencias, visiones, concepciones y discursos que se asocian con modelos pedagógicos. Son las decisiones pedagógicas las que delinean los procesos formativos, de ahí su importancia, ya que una pedagogía pertinente "permite mejorar la enseñanza y cualificar la educación del pueblo colombiano para avanzar por la senda del desarrollo económico y social que necesita el país" (Flórez, 2006: 66). No obstante, la apreciación ciudadana sobre la calidad de los procesos pedagógicos puede estar mediada por la subjetividad, por ejemplo de una experiencia escolar pasada del ciudadano o por la relación que este establezca con docentes cercanos, siendo poco objetiva la información que ofrezcan sobre este tema específico.

\section{3.- Resultados del modelo de regresión sobre la percepción ciudadana en materia educativa en Colombia.}

A partir del procedimiento referido en el apartado metodológico, se obtuvo un modelo de regresión logística con las siguientes variables: la percepción sobre el cumplimiento del derecho a la educación; la percepción de la inversión en educación, opinión sobre la evolución del país; la percepción sobre la utilidad de las pruebas de estado; los niveles

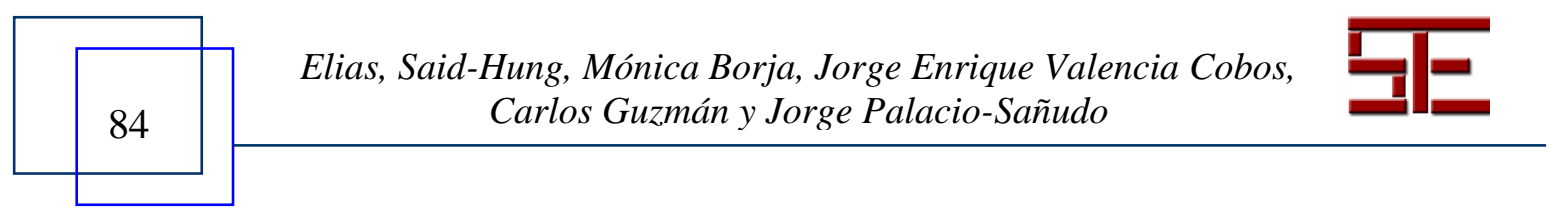




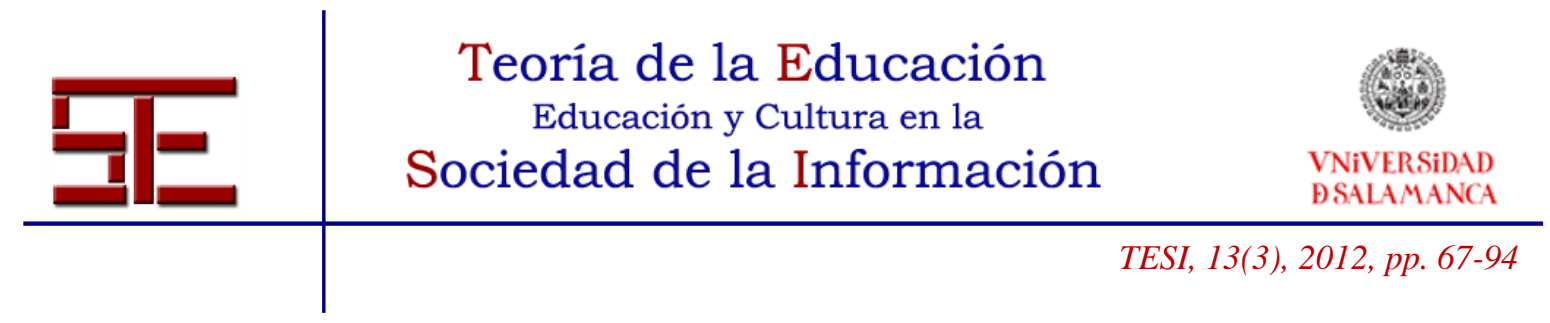

de satisfacción con la gestión de la Secretaría de Educación de la ciudad; la actualización de los profesores; la dotación de los colegios; la administración de los colegios, y el servicio de alimentación escolar.

La Tabla 5 presenta los resultados del modelo final. En esta se incluyen los coeficientes de regresión (B), el error estándar de estimación (E.T.), el valor del estadístico W de Wald, con los grados de libertad (g.l.) y su nivel de significancia (Sig.) y el Riesgo (Exp (B)) de cada variable con sus respectivos intervalos de confianza.

Tabla 5: Resultados del modelo de regresión logística

\begin{tabular}{|c|c|c|c|c|c|c|c|c|}
\hline \multirow{2}{*}{ Variables en la ecuación } & \multirow{2}{*}{ B } & \multirow{2}{*}{ E.T. } & \multirow{2}{*}{ Wald } & \multirow{2}{*}{ gl } & \multirow{2}{*}{ Sig. } & \multirow{2}{*}{$\begin{array}{c}\operatorname{Exp} \\
(\mathbf{B})\end{array}$} & \multicolumn{2}{|c|}{$\begin{array}{l}\text { I.C. } 95 \% \text { para } \\
\operatorname{EXP}(B)\end{array}$} \\
\hline & & & & & & & Inferior & Superior \\
\hline La administración de los colegios & 625 & 280 & 4,987 & 1 & ,026 & 1,867 & 1,079 & 3,231 \\
\hline La actualización de los profesores & 1,295 & ,280 & 21,378 & 1 &, 000 & 3,650 & 2,108 & 6,318 \\
\hline La dotación de los colegios & ,631 & ,262 & 5,786 & 1 & ,016 & 1,879 & 1,124 & 3,142 \\
\hline La alimentación escolar & ,747 & ,264 & 7,971 & 1 &, 005 & 2,110 & 1,257 & 3,544 \\
\hline $\begin{array}{l}\text { ¿A Usted y su familia le están } \\
\text { garantizando el derecho a la educación? }\end{array}$ & 1,107 & ,233 & 22,630 & 1 &, 000 & 3,024 & 1,917 & 4,771 \\
\hline $\begin{array}{l}\text { ¿Usted cree que la educación en } \\
\text { nuestro país ha mejorado, se mantiene } \\
\text { igual o ha empeorado en los últimos } \\
\text { diez años? }\end{array}$ & & & 18,206 & 2 &, 000 & & & \\
\hline Ha mejorado & 1,114 & ,261 & 18,185 & 1 & ,000 & 3,048 & 1,826 & 5,087 \\
\hline Se mantiene igual & ,562 & ,300 & 3,505 & 1 & ,061 & 1,754 & ,974 & 3,157 \\
\hline $\begin{array}{l}\text { Cómo califica la gestión que ha hecho } \\
\text { hasta ahora la Secretaría de Educación } \\
\text { de esta ciudad }\end{array}$ & ,477 & ,239 & 3,988 & 1 & ,046 & 1,611 & 1,009 & 2,571 \\
\hline $\begin{array}{l}\text { ¿Usted cree que su ciudad está } \\
\text { invirtiendo lo que se requiere para el } \\
\text { tema de educación? }\end{array}$ & ,932 &, 325 & 8,232 & 1 & ,004 & 2,540 & 1,344 & 4,802 \\
\hline
\end{tabular}

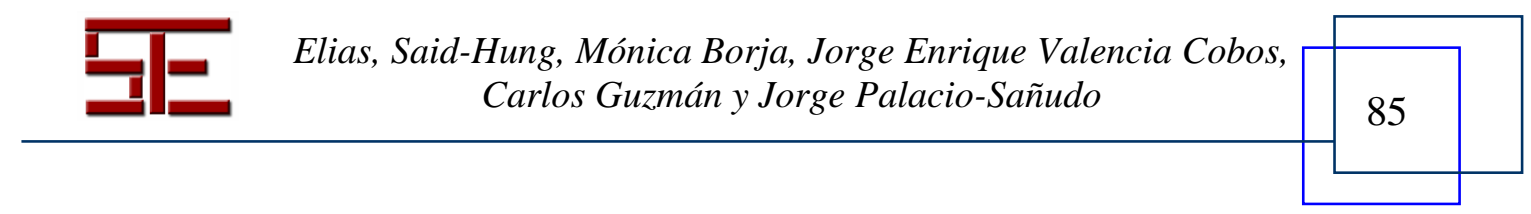




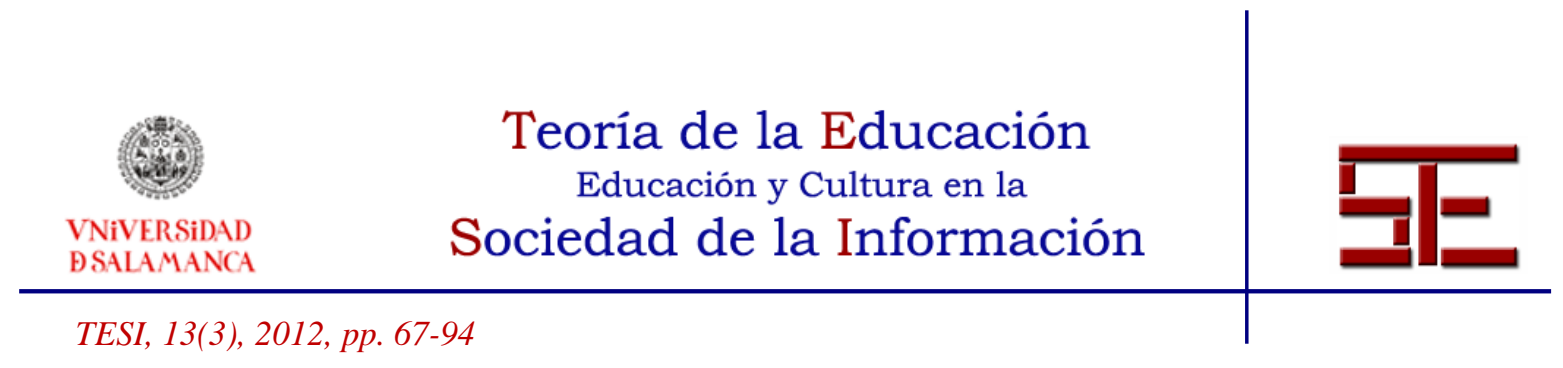

\begin{tabular}{|c|c|c|c|c|c|c|c|c|}
\hline $\begin{array}{l}\text { ¿Usted cree que este tipo de pruebas } \\
\text { (ICFES, SABER; ECAES) sirven para } \\
\text { determinar la calidad de la educación } \\
\text { en Colombia? }\end{array}$ & ,666 &, 240 & 7,673 & 1 & ,006 & 1,947 & 1,215 & 3,118 \\
\hline Constante & $-3,707$ & ,399 & 86,457 & 1 & ,000 & ,025 & & \\
\hline
\end{tabular}

Fuente: Elaborada por los autores.

Dato: $\mathrm{N}=1640$.

La estimación del Riesgo "Odds ratio" (Exp (B)) obtenida para cada variable informa sobre los detalles de la relación de cada variable con la satisfacción o no con el sistema educativo. Así pues, los resultados mostrados en la Tabla 5 nos muestran como la probabilidad de estar satisfecho con el sistema educativo colombiano es:

- $\quad$ 1,867 veces más probable en aquellos encuestados que se encuentran satisfechos con la administración de los colegios.

- 3,650 veces más probable entre los encuestados que se encuentran satisfechos con la actualización de los profesores.

- 1,879 veces más probable entre los encuestados que se encuentran satisfechos con la dotación de los colegios.

- 2,110 veces más probable entre los encuestados que se encuentran satisfechos con el servicio de alimentación escolar.

Todo lo anterior, en comparación con los encuestados que manifestaron estar insatisfechos con cada una de estas variables, siendo esta la categoría de referencia para este grupo de variables.

Por otra parte encontramos (Tabla 5) que la probabilidad de estar satisfecho con la situación educativa en Colombia es:

- 3,024 veces más probable entre los encuestados que consideran que se está garantizando el derecho a la educación en el país, frente a quienes no.

- 2,540 veces más probable entre los encuestados que manifestaron que en su cuidad se está invirtiendo lo suficiente en educación, frente a los que no (categoría de referencia).

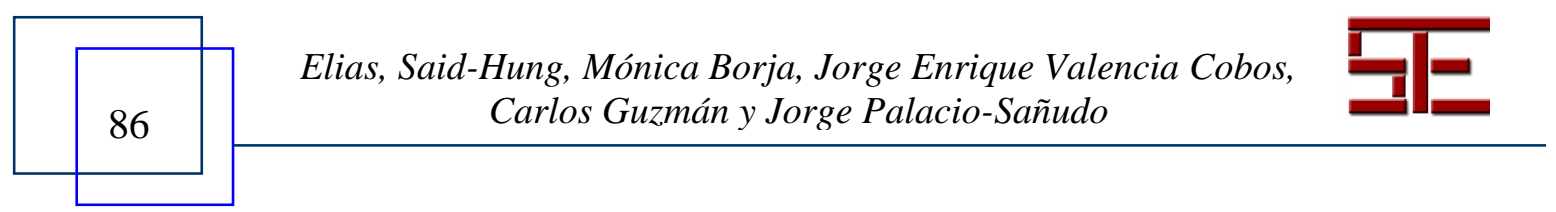




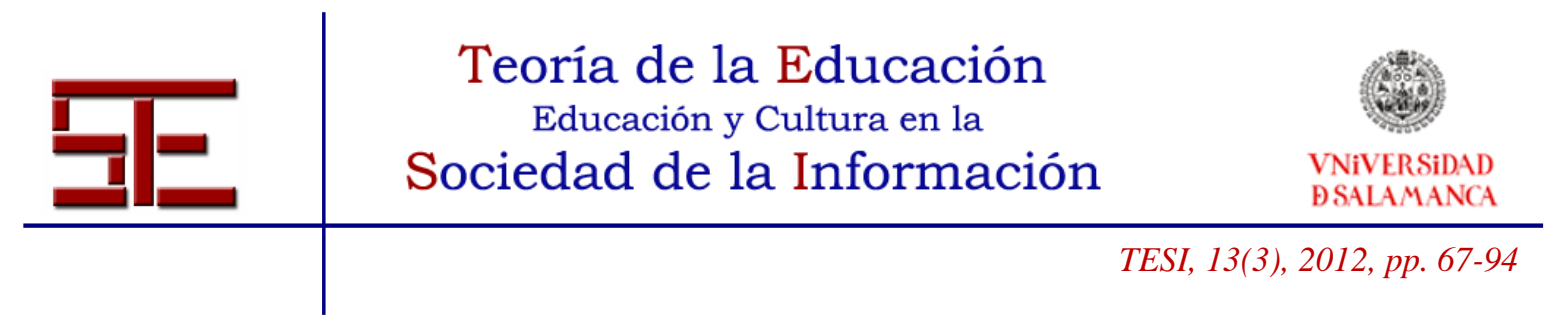

- 1,611 veces más probable entre los encuestados que consideran como buena la gestión de las Secretarías de Educación de su ciudad frente a quienes no.

Finalmente encontramos que la probabilidad de estar satisfecho con el sistema educativo colombiano es:

- 1,947 veces más probable en aquellos encuestados que consideran que las pruebas de estado sirven para determinar la calidad de la educación comparados con quienes no.

- 3,048 veces más probable entre los encuestados que consideran que la situación del país ha mejorado, teniendo como referencia a quienes consideran que ha empeorado. En este sentido, la comparación de esta última categoría con quienes consideran que la situación del país sigue igual o no ha variado, no es significativa.

La evaluación de la bondad de ajuste del modelo se realizó a través de la deviación (2LL), el R2 de Cox-Snell, el R2 Nagelkerke y la prueba de Hosmer y Lemeshow. De la Tabla 6 podemos resaltar que el coeficiente R2 de Nagelkerke indica que el 49,3\% de la variación en la satisfacción con el sistema educativo se encuentra explicado por el modelo, en los rangos aceptados en otros estudios, llevados a cabo por Cassaus (2000); Rodríguez-Ayán (2005), Campo-Arias, et al., (2005), entre otros, cuyos valores para este indicador se situaron entre $27,5 \%$ y 50,4\%. Adicionalmente, debe agregarse que el porcentaje de coincidencias obtenido de la tabla de clasificación fue de $84,3 \%$ lo que indica que el modelo presenta una buena capacidad de predicción.

Tabla 6: Pruebas de validez y ajuste

\begin{tabular}{cccccc}
\hline \multicolumn{2}{c}{ Medidas de Validez } & \multicolumn{2}{c}{ Prueba de Hosmer y Lemeshow } \\
$-2 \log$ de la verosimilitud & $\begin{array}{c}\text { R cuadrado de } \\
\text { Cox y Snell }\end{array}$ & $\begin{array}{c}\text { R cuadrado de } \\
\text { Nagelkerke }\end{array}$ & Chi cuadrado & gl & Sig. \\
\hline $516,855^{\text {a }}$ &, 337 &, 493 & 3,261 & 8 &, 917 \\
\hline
\end{tabular}

Fuente: Elaborada por los autores.

Dato: $\quad \mathrm{N}=1640$.

a. La estimación ha finalizado en el número de iteración 6 porque las estimaciones de los parámetros han cambiado en menos de ,001.

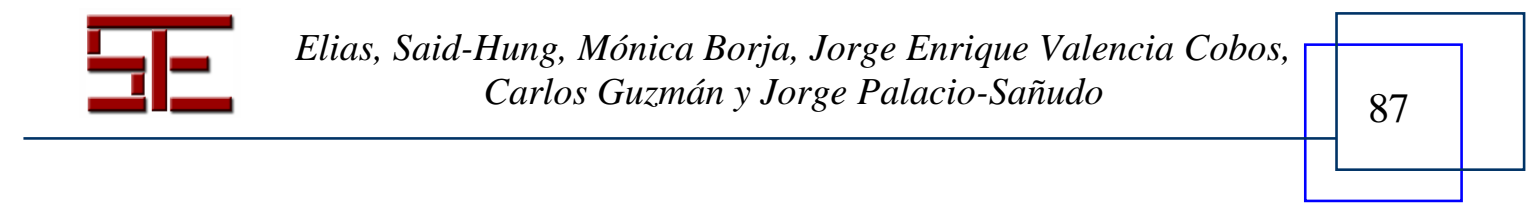




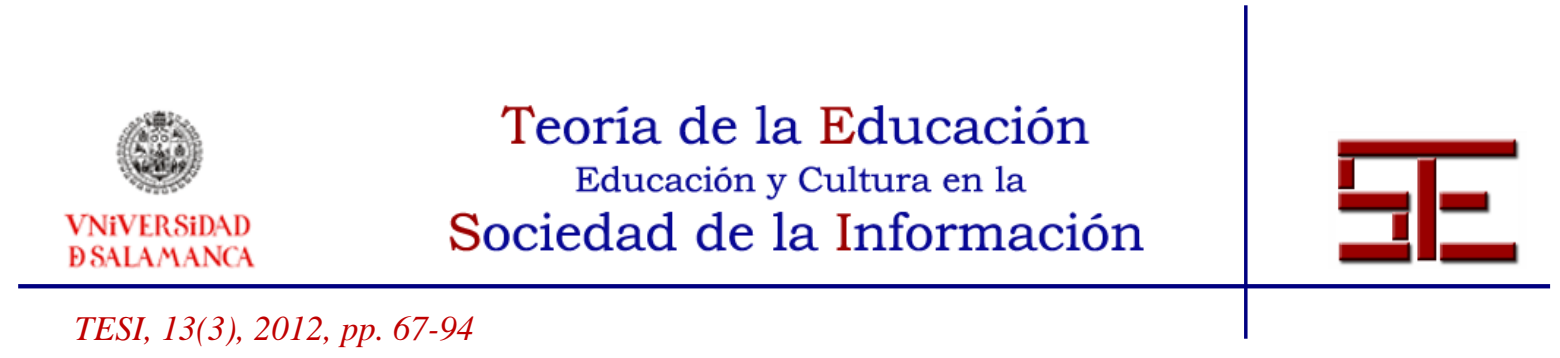

\section{4.- DISCUSIÓN, CONCLUSIONES Y PERSPECTIVA}

Como resultado del análisis, se lograron identificar dos grupos de factores que determinaron la percepción sobre el sistema educativo colombiano, el primero da cuenta de factores asociados a la escuela y la dinámica escolar, el segundo de aspectos normativos y de gestión pública.

A nivel de la escuela, tuvieron mayor influencia sobre la formación de la percepción del sistema educativo en los 1.690 encuestados: la administración de los colegios, la actualización de los profesores, la dotación de los colegios, el servicio de alimentación escolar y la percepción sobre la utilidad de las pruebas de estado. De la valoración de estos elementos se deduce la influencia multidimensional (social, cultural, formativa) que afecta la construcción colectiva del concepto de calidad educativa lo que coincide con el enfoque integral centrado en la armonización del conjunto de actores, procesos, medios, recursos puesto al servicio de las metas educativas, lo cual se aleja del concepto clásico de escuelas eficaces en el cual la calidad de un centro y de la educación que ofrece depende esencialmente del recurso humano que orienta los procesos formativos (Gimeno y Pérez, 1997; Mañú, 2005; Bazarra, Casanova \& García, 2007). En esta conceptualización intervienen la labor docente, la gestión administrativa, la infraestructura, el apoyo nutricional y los resultados académicos, acompañado de un alto nivel de satisfacción presentado, no solo en los elementos descritos en el modelo, sino en general con todo el sistema educativo.

Sobre este último punto, debe destacarse que las percepciones ciudadanas se nutren tanto de elementos objetivos como subjetivos, por lo tanto es pertinente preguntarse, ¿qué tan ajustada es esta percepción de satisfacción a la realidad de la calidad educativa en Colombia? Como señalamos en el apartado conceptual de este artículo, tanto a nivel de resultados en las pruebas de rendimientos de los alumnos como en la articulación de políticas y acciones orientadas al fortalecimiento de la Calidad de la Educación en Colombia, el panorama educativo en Colombia es crítico y divergente con la percepción favorable que tienen los ciudadanos en este país, sobre este tema. Sin embargo, la percepción ciudadana da en el blanco de uno de los factores más importantes en el mejoramiento de la calidad educativa: la actualización de los profesores. Lo aquí expuesto, además de confirmar lo señalado por Vélez, Schiefelbein y Valenzuela (1996) y Lora (2008), entre otros investigadores del área, contribuye a realizar dos afirmaciones: 1) el alto valor de los estudios de percepción ciudadana, al momento de diseñar políticas públicas en materia educativa en América Latina y Colombia, nuestro

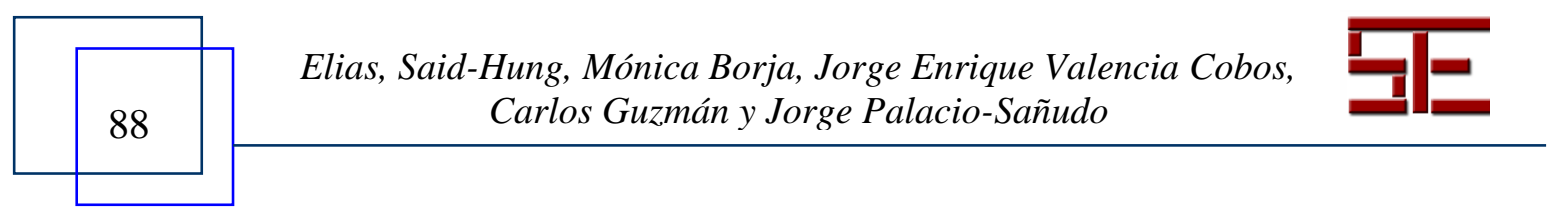




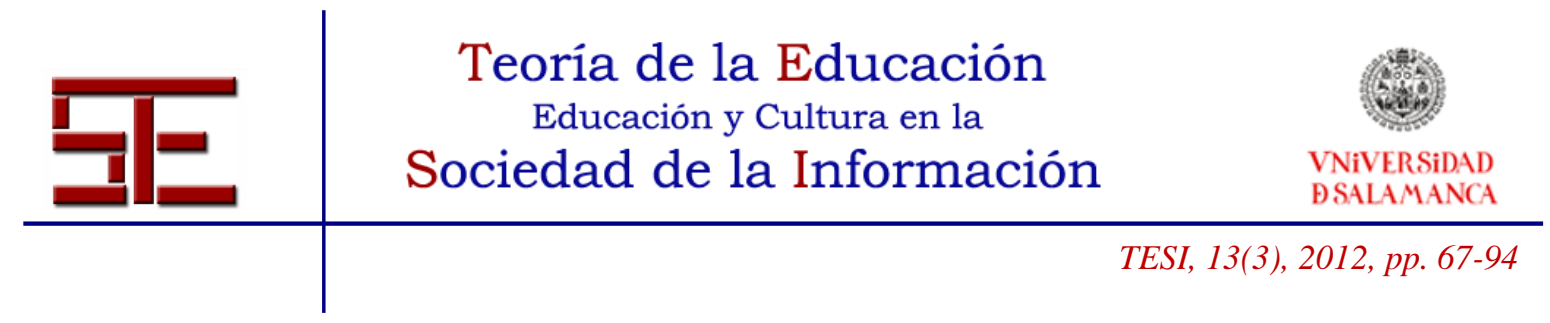

caso de estudio, al permitir estos un mayor conocimiento de los factores de carácter multidimensional (social, cultural, formativo, entre otros) que inciden en la visión que tienen los ciudadanos del derecho y calidad de la educación; y 2) la necesidad de dar el llamado de atención a los entes públicos responsables en el diseño de estas políticas, al menos en el caso colombiano, de la necesidad de ampliar los temas esenciales que permitan mejorar al sector educativo a nivel nacional; para lo que se necesitaría no solo considerar como ejes centrales del diseño de políticas la cobertura, rendimiento académico, la pertinencia de los programas curriculares o el aumento de la capacitación técnica en Colombia; sino también, se requiere, de acuerdo con los datos analizados en este artículo, centrarse en factores asociados a la escuela, la dinámica escolar e inclusión desde estos escenarios de enseñanza, especial en lo que se refiere a: cómo se administran los colegios, la actualización de los profesores, la dotación de estas instituciones y el servicio de alimentación escolar; y en factores vinculados con el marco normativo y de gestión pública, relacionada con el sector educativo (como por ejemplo, la garantía al derecho a la educación y la eficiencia en la gestión pública de los actores políticos locales).

\section{5.- BIBLIOGRAFÍA}

Arancibia, V. (1994). La educación en Chile: percepciones de la opinión pública y de expertos. Estudios Públicos, 54, 125-551.

Aron, R. (1977). Aspects sociologiques des notions de quantité et qualité en fait d'éducation. París: UNESCO.

Aziz, A., Canales, A. et al. (2001). Calidad de la Educación. Bogotá: Observatorio Ciudadano de la Educación.

Banco Mundial (2008). La calidad de la educación en Colombia: un análisis y algunas opciones para un programa de política. Washington: Autor.

Bazarra, L. Casanova, O. \& García J. (2007). Profesores, Alumnos, Familias. 7 pasos para un nuevo modelo de escuela. Madrid: Narcea.

Bourdieu, P. (1973). Cultural Reproduction and Social Reproduction. En R. Brown (Ed.), Knowledge, Education, and Social Change: Papers in the Sociology of Education (71-112). London: Tavistock.

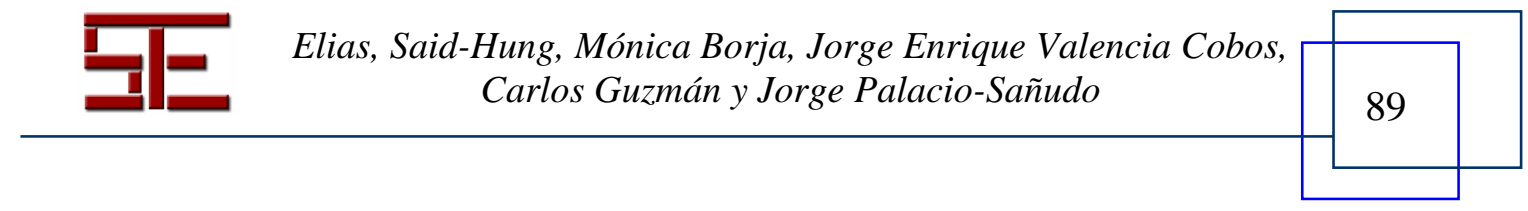




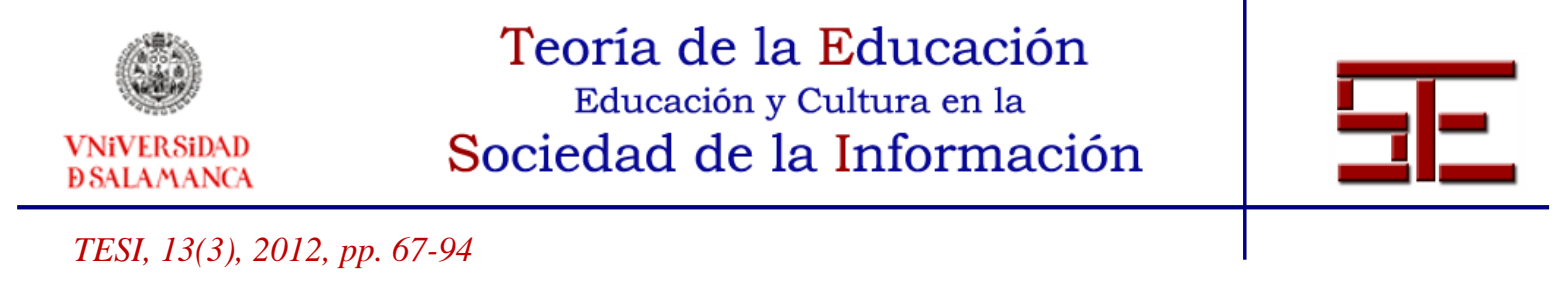

- (2000). Cuestiones de Sociología. Madrid: Ediciones AKAL.

Brookover, W. B. et al. (1979). School social systems and student achievement: Schools can make a difference. New York: Praeger.

Brunner, J. (2000). Globalización y el futuro de la educación: tendencias, desafíos, estrategias. Extraído el 10 de mayo, 2011, de http://www.schwartzman.org.br/simon/delphi/pdf/brunner.pdf.

Campo-Arias, A. et al. (2005). Percepción de rendimiento académico y síntomas depresivos en estudiantes de media vocacional de Bucaramanga, Colombia. Arch. Pediatr. Urug. 76, 21-26.

Cassaus, J. et al. (2000). Primer estudio internacional comparativo. Sobre lenguaje, matemática y factores asociados, para alumnos del tercer y cuarto grado de la educación básica. Santiago de Chile: Laboratorio Latinoamericano de Evaluación de la Calidad de la Educación.

Castilla, M. (2005). Funciones del profesorado en la sociedad actual. En I. Cabello \& F. de Vicente (Eds.), El profesorado y los retos del sistema educativo actual (pp. 133184). Madrid: Ministerio de Educación y Ciencia de España.

CEPAL, OEI \& Secretaría General Iberoamericana (2010). 2021, Metas Educativas. La educación que queremos para la generación de los Bicentenarios. Madrid: Autores.

CEPAL \& UNESCO (2005). Invertir mejor para invertir más. Financiamiento y gestión de la educación en América Latina y el Caribe. Santiago de Chile: Naciones Unidas.

Charlot, B. (2008). La relación con el saber, formación de maestros y profesores, educación y globalización. Cuestiones para la edución de hoy. Montevideo: Ed Trilce.

Creemers, B. (1994). The effective Classroom. London: Cassell.

Departamento de Planeación Nacional (2003). Plan Nacional de Desarrollo 2002-2006. Hacia un Estado Comunitario. Bogotá: Autor.

- (2007). Plan Nacional de Desarrollo 2006-2010. Estado Comunitario: Desarrollo para Todos. Bogotá: Autor.

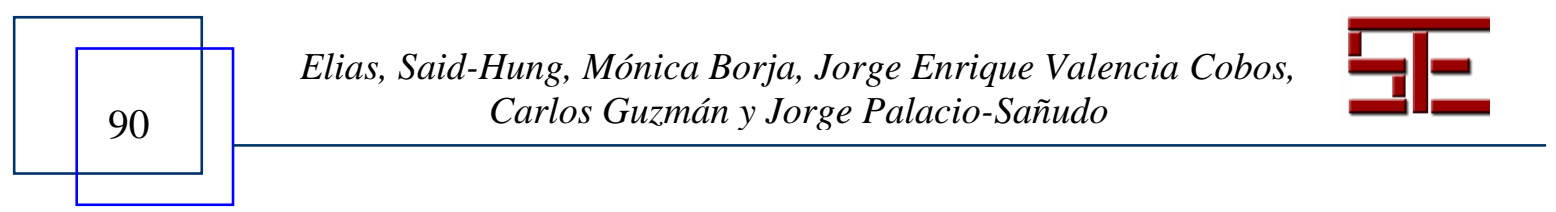




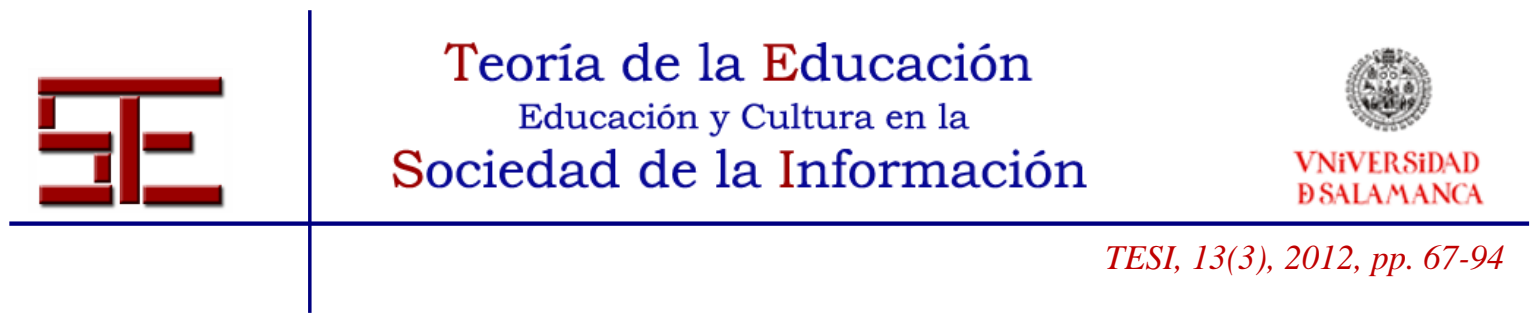

- (2011). Plan Nacional de Desarrollo 2010-2014. Prosperidad para Todos. Bogotá: Autor.

Edmonds, R. (1982). Programs of school improvement: An overview. Educational Leadership, 40, 4-11.

Elacqua, G. \& Fábrega, R. (2006). El consumidor de la educación: el actor olvidado de la libre elección de escuelas en Chile. En PREAL (Ed.), Educación y Brechas de Equidad en América Latina. Tomo II (pp. 353-398). Washington: PREAL.

Flórez, R. (2006). Hacia una nueva cultura educativa. Revista Educación y Pedagogía, $38,61-69$.

Gil-Pérez, D. \& Vilches, A. (2005). La participación en el debate educativo como deber ciudadano. Comentarios y sugerencias en torno al documento "Un Educación de Calidad para todos y entre todos". Revista Eureka sobre Enseñanza y Divulgación de las Ciencias, 2, 251-268.

Gimeno, J. \& Pérez, A. (1997). Comprender y Transformar la Enseñanza. Madrid: Morata.

Herrera, M. \& López, M. (1996). Estudio comparativo de Fe y Alegría y Escuelas Oficiales (nacionales y municipales). Caracas: Banco Mundial- CIDE.

ICFES (2010). Colombia en Pisa 2009. Síntesis de resultados. Bogotá: Autor.

Lerma, C. (2007). El derecho a la educación en Colombia. Bogotá: FLAPE.

Lora, E. (2008). Calidad de Vida. Más allá de los hechos. México: Banco Interamericano de Desarrollo y Fondo de Cultura Económica.

Mañú, J. (2005). Equipos directivos para centros de calidad. Madrid: Ed. RIALP.

Marinho, M. (2007). El eslabón perdido entre educación y empleo. Análisis sobre las percepciones de los jóvenes urbanos de escasos recursos en Chile. Políticas Sociales, 137. Extraído el 10 diciembre, 2010, de http://www.eclac.org/publicaciones/xml/3/32663/sps137_lcl2783.pdf.

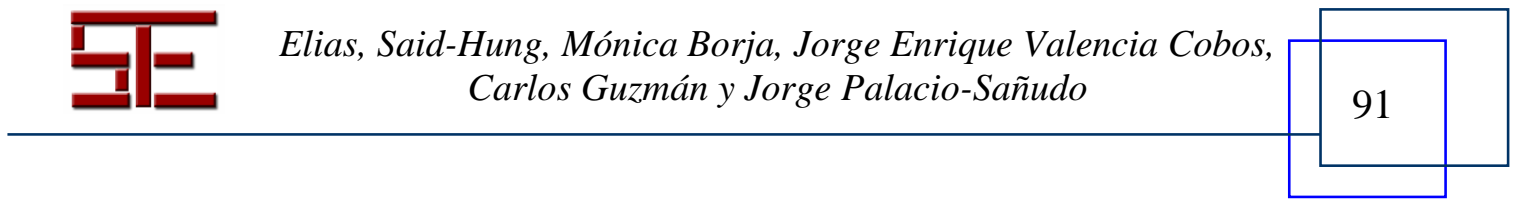




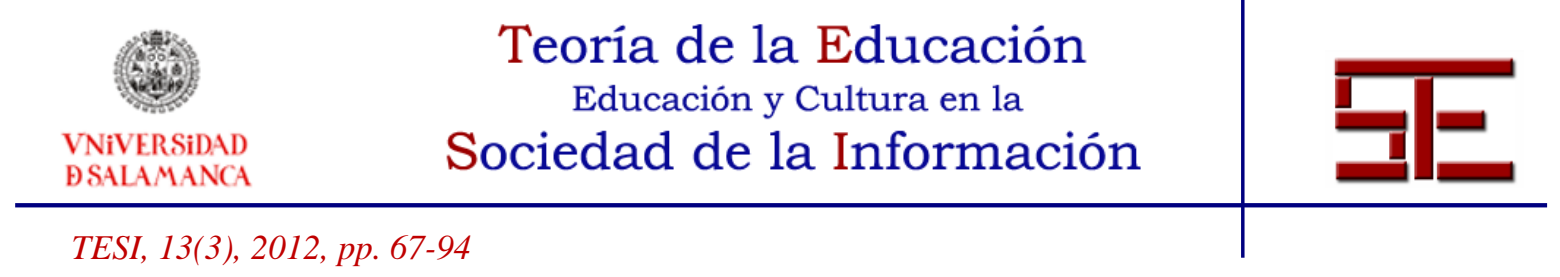

Marquès, P. (2002). Calidad e innovación educativa en los Centros. Extraído el 11 de julio, 2011, de http://www.peremarques.net/calida2.htm.

Ministerio de Educación Nacional de Colombia (2007). Boletín informativo Plan Decenal de Educación 2006-2015. Extraído el 10 noviembre, 2010, de http://menweb.mineducacion.gov.co/educacion_superior/plandecenal/004.htm.

Mizala, A. \& Urquiola, M. (2007). Socioeconomic status or noise? Tradeoffs in the generation of school quality information. Journal of Development Economics, $84,61-75$.

Mortimore, P. et al. (1998). School Matters: The juniors years. London: Open Book. Naciones Unidas (1948). Declaración Universal de los Derechos Humanos. Extraído el 2 de noviembre, 2010, de http://www.un.org/es/documents/udhr/index.shtml\#a26.

- (2010). Objetivos de Desarrollo del Milenio. Informe 2010. Extraído el 2 de noviembre, 2010, de http://www.un.org/es/comun/docs/?path=/spanish/millenniumgoals/pdf/MDG_R eport_2010_SP.pdf.

OCDE (1995). Análisis del panorama educativo. Indicadores de la OCDE. París: Mundi-Prensa.

OEI (2010). 2021, Metas Educativas. La educación que queremos para la generación de los Bicentenarios. Madrid: CEPAL, OEI y Secretaría General Iberoamericana.

PREAL (2005). Cantidad sin calidad. Informe del Progreso Educativo en América Latina del Programa de Promoción de la Reforma Educativa en América Latina y el Caribe. Madrid: Organización de Estados Iberoamericanos. Extraído el 15 de febrero, 2011, de http://www.oei.es/quipu/Informe_preal2006.pdf.

Purkey, S. \& Smith, M. (1983). Effective Schools: A review. The Elementary School Journal, 83, 412-452.

Rodríguez, N. (2000). Gestión escolar y calidad de la enseñanza. Educere, 4, 39-46.

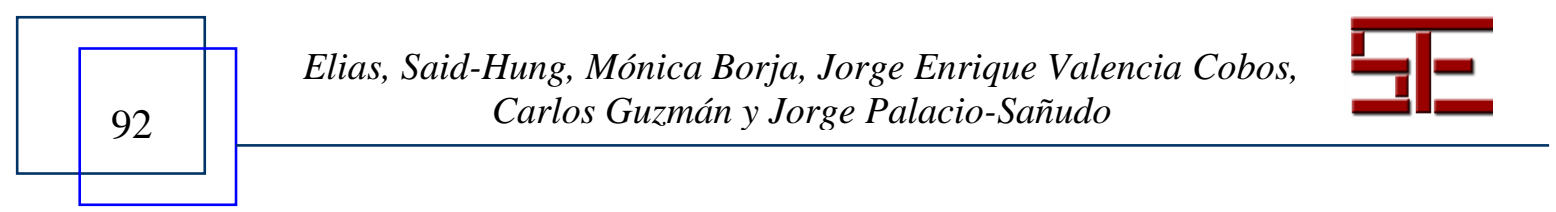




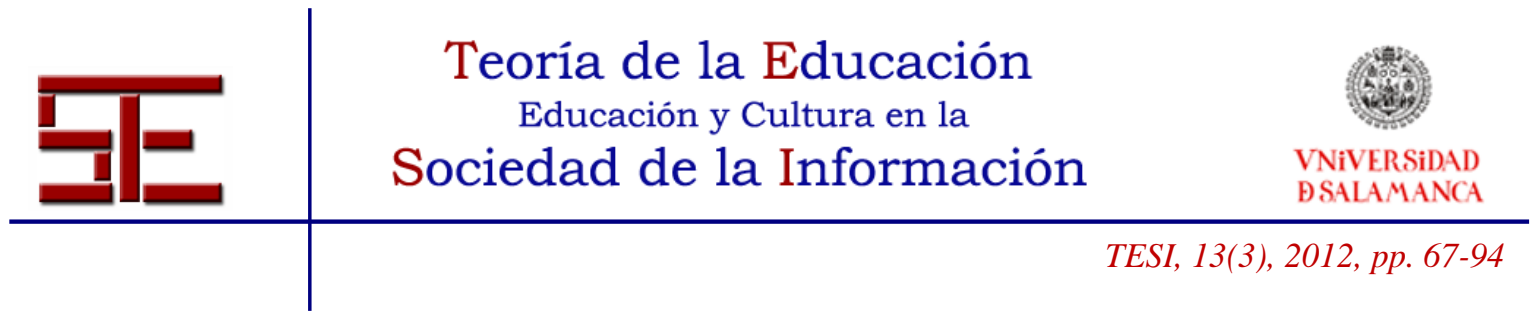

Rodríguez-Ayán, M. (2005). La perspectiva estudiantil sobre el desempeño del profesor: un modelo de regresión logística ordinal. Revista Electrónica de Metodología Aplicada, 10, 1-13.

Sarmiento, A. (2010). Situación de la Educación en Colombia. Preescolar, básica, media y superior. Bogotá: Educación Compromiso de Todos.

Tiana, A. \& Santángelo, H. (1994). Evaluación de la Calidad de la Educación. Revista Iberoamericana de Educación, 10. Extraído el 15 de junio, 2011, de http://www.oei.org.co/oeivirt/fp/cuad1a04.htm.

Tomasevski, K. (2004). Los derechos económicos, sociales y culturales: El derecho a la educación. Extraído el 11 de agosto, 2011, de http://www.observatoriopoliticasocial.org/images/PDF/Biblioteca/biblioteca_2010/ON U_docs/Informes_relatores/Educacion/2004_informe_de_la\%20relatora_especial_sobre _el_derecho_a_la_educacion.pdf.

Toral, R. (2003, julio 1). Calidad en la educación. Odiseo, revista electrónica de pedagogía 1. Extraído el 12 de febrero, 2010 de http://www.odiseo.com.mx/2003/07/print/02toral_calidad.htm.

Vega, A. (2008). Sociedad del conocimiento y calidad de la educación. Cuadernos de Docencia Universitaria, 1, 129-135.

Vega, E. \& Petrow, J. (2007). Raising student achievement in Latin America: the challenge for the 21th century. Washington: Latin American Development Forum.

Vélez, E., Schiefelbein, E. \& Valenzuela, J. (1996). Factores que afectan el rendimiento académico en la educación primaria. Extraído el 10 de noviembre, 2010, de http://www.oei.es/calidad2/Velezd.PDF.

Willms, D., Sommers, A. \& Pardo, C. (2001). Primer estudio internacional comparativo sobre lenguaje, matemática y factores asociados, para alumnos del tercer y cuarto grado de la educación básica. Informe Técnico. Santiago: UNESCO.

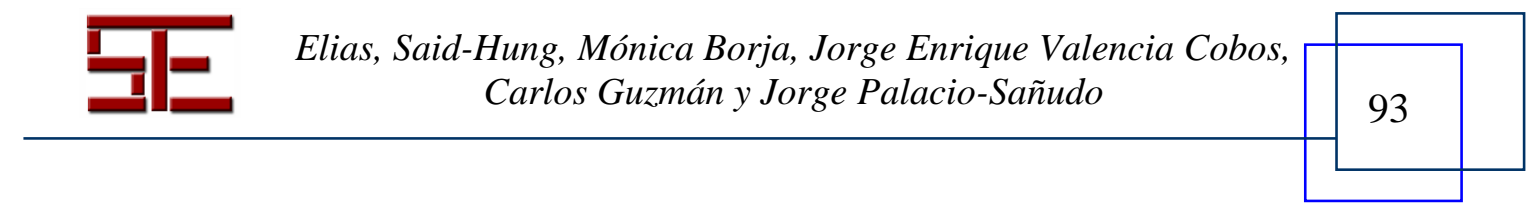




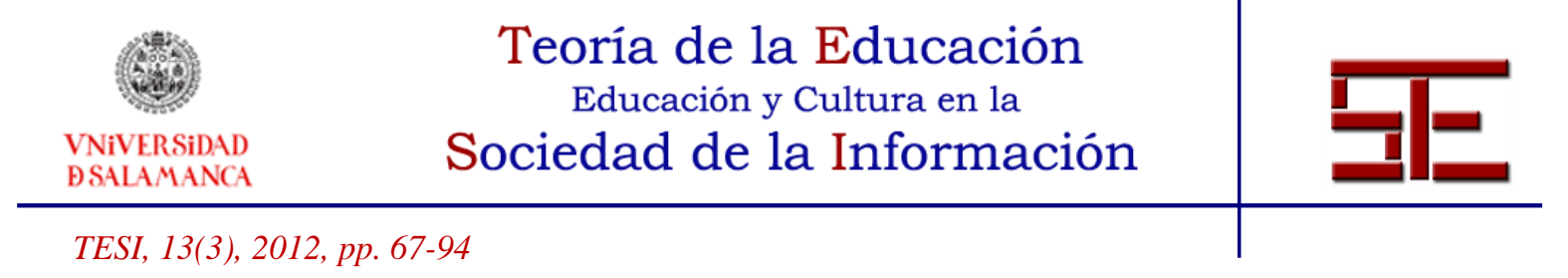

Para citar el presente artículo puede utilizar la siguiente referencia:

Said-Hung, E., Borjas, M., Valencia Cobos, J., Palacio-Sañudo, J. E. y Guzmán, C. (2012). Determinantes sobre la percepción ciudadana de la educación en Colombia. Revista Teoría de la Educación: Educación y Cultura en la Sociedad de la Información. 13(3), 67-93 [Fecha de consulta: dd/mm/aaaa].

http://campus.usal.es/ revistas_trabajo/index.php/revistatesi/article/view/9220/9480

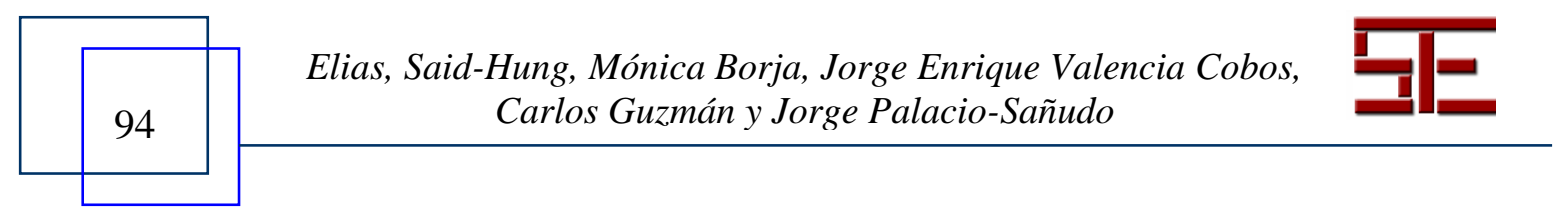

